\title{
THE ANNUAL MEETING IN HOUSTON
}

The sixty-second Annual Meeting of the American Mathematical Society was held at The Rice Institute, Houston, Texas, on Tuesday, Wednesday, and Thursday, December 27-29, 1955, in conjunction with the Annual Meeting of the Mathematical Association of America on Friday, December 30. The attendance was nearly 400, including 265 members of the Society.

The twenty-ninth Josiah Willard Gibbs Lecture was delivered by Professor J. E. Mayer of the University of Chicago on Tuesday at 8:00 P.M. Professor Mayer's lecture was entitled The structure of simple fluids. Presiding officer at the session, held in the Auditorium of the Prudential Insurance Company, was President William V. Houston of The Rice Institute.

The Presidential Address was delivered by Professor G. T. Whyburn of the University of Virginia on Wednesday at 10:30 A.M. with Professor R. L. Wilder presiding. Professor Whyburn's topic was Topological analysis. Professor R. L. Moore was in the audience and Professor Wilder took the opportunity to pay him appropriate tribute.

By invitation of the Committee to Select Hour Speakers for Annual and Summer Meetings, Professor S. S. Chern of the University of Chicago addressed the Society on Complex vector bundles. Professor Deane Montgomery presided at the lecture.

There were a total of fourteen sessions at which contributed papers were presented. Presiding officers at these various sessions were Professors H. M. MacNeille, J. J. Gergen, W. T. Scott, F. B. Jones, E. F. Beckenbach, L. C. Young, R. H. Bing, H. F. Bohnenblust, H. E. Bray, C. B. Allendoerfer, R. M. Thrall, G. R. MacLane, Dr. John Todd and Mr. Michael Goldberg.

There was a banquet in The Rice Institute Commons on Wednesday at 6:30 P.M., a banquet unique in the recent history of the Society both for the caliber of the food and the quality of the speeches. Professor Wilder, the President of the Society, acted as Toastmaster and introduced President William V. Houston of The Rice Institute. President Houston was followed by President W. L. Duren, Jr. of the Mathematical Association of America. A resolution of thanks to The Rice Institute was read by Dr. L. W. Cohen of the National Science Foundation.

On Thursday afternoon there was a trip to a refinery of the Humble Oil and Refining Company and a number of the visiting mathemati- 
cians got a glimpse of the manufacturing aspect of the oil business. In the evening there was a concert by the Lyric Art String Quartet.

The ladies of The Rice Institute Department of Mathematics presided daily at teas served for the mathematicians in the lounge of the Faculty Club.

The Council met on Wednesday afternoon, December 28, 1955.

The Secretary announced the election of the following forty persons to ordinary membership in the Society:

Mrs. Fortunata Vitanza Altmayer, Ford Instrument Co., Long Island City, N. Y.; Mr. William Moses Ashley, Douglas Aircraft Company, Santa Monica, California;

Dr. Roger Harris Bender, Datamatic Corporation, Newton Highlands, Massachusetts;

Professor Joseph Buffington Roberts, Reed College;

Professor Donald Lyman Burkholder, University of Illinois;

Mr. William Virgil Caldwell, University of Michigan;

Mr. John Cocke, Duke University;

Dr. Thomas H. Crowley, Bell Telephone Laboratories, Murray Hill, New Jersey;

Dr. John Warren Dettman, Bell Telephone Laboratories, Whippany, New Jersey;

Mr. Lionel David Dureau, Tulane University;

Dr. Walter James Feeney, Weston College;

Dr. Bobby J. Hollingsworth, United Gas Corporation, Shreveport, Louisiana;

Dr. James Lucien Howland, Computing Devices of Canada, Ltd., Ottawa, Ontario, Canada;

Mr. Robert Shepard Johnson, University of Pennsylvania;

Dr. John Killeen, Bell Telephone Laboratories, Murray Hill, New Jersey;

Mr. Naoki Kimura, Tokyo Institute of Technology;

Dr. Vernon Albert Kramer, University of California, Riverside;

Mr. James Lanier Knott, Miles College;

Professor Lester E. Laird, Kansas State Teachers College;

Mr. Muniswamyroddy Lakshmanan, Maryland College, Liberia, West Africa;

Mr. Henry Curt Lefkovits, Shell Development Co., Houston, Texas, and Rice Institute;

Mr. Harry W. Lew, Hughes Aircraft Company, Culver City, California;

Professor Carlos B. de Lyra, University de Sao Paulo and Instituto de Matematica Pura e Aplicada, Brazil;

Mr. Stanley Walter Malinowski, Moore School, University of Pennsylvania;

Dr. Michael Anthony Martino, National Security Agency, Washington, D. C.;

Professor Robert T. McLean, College of Steubenville;

Dr. Trevor James McMinn, University of California, Berkeley;

Mr. Fritz Walter Mezger, General Electric Company, Cincinnati, Ohio;

Professor Taro Morishima, Tokyo College of Science, Tokyo, Japan;

Sister Irene Morvan, Annhurst College;

Professor William J. Pervin, University of Pittsburgh;

Mr. Robert Charles Pfeilsticker, Frankford Arsenal, Philadelphia, Pennsylvania;

Dr. Bayard Rankin, Massachusetts Institute of Technology;

Mr. Neal Jules Rothman, Burroughs Corporation, Paoli, Pennsylvania;

Professor Robert Winter Royston, Washington and Lee University;

Mr. Joseph Julius Schoderbek, Lockheed Aircraft Corporation, Van Nuys, California;

Professor Ivar Stakgold, Harvard University; 
Mr. V. Thillainayagam, Annamalai University, Annamalainagar, South India;

Mr. Frederick Leonard Zarnfaller, Olin Mathieson Chemical Corporation, New Haven, Connecticut;

Mr. Fred Selwyn Zusman, John Hopkins University.

It was reported that the following forty-five persons had been elected to membership on nomination of institutional members as indicated:

University of British Columbia: Mr. George Elliot Cross.

The University of Cincinnati: Mr. Roger Chalkley.

Columbia University: Mr. Joseph Francis Manogue, Mr. Lee Paul Neuwirth, Miss Helen Lynn Paisner, Mr. Jyri Erkki Paloheimo, Mr. John Wolfgang Smith, and Mr. Joe Fred Traub.

Cornell University: Mr. David Norman Freeman, Mr. Benoit Vincent Lachapelle, Mr. Shing-Ming Lee, Mr. George Fitzgerald Lowerre, Mr. Richard Felix McCoart, Mr. Edward Norman, and Miss Marion Ilse Walter.

University of Georgia: Mr. James J. Andrews.

Iowa State College: Mr. William Duane Montgomery.

University of Kansas: Mr. Robert Brainard Buckley.

Massachusetts Institute of Technology: Mr. William Bernard Houston, Jr., Mr. Millard Wallace Johnson, Mr. Robert Richard Dingle Kemp, Mr. Donald L. Kreider, Mr. Albert Louis Rabenstein, Mr. Anthony Ralston, and Dr. Leonard Roberts.

University of Michigan: Mr. Michael Barr, Mr. James Oliver Brooks, Mr. Ross Lee Finney, III, Mr. Gordon McCrea Fisher, Mr. Carlos Imaz, Mr. Richard Patterson Jerrard, Mr. Howard Earl Reinhardt, Mr. Roger Wolcott Richardson, Jr., Mr. Neal McCalla Speake, Mr. Joseph Gail Stampfli, and Mr. William Blauvelt Woolf.

University of New Hampshire: Mr. Bruce Cale McQuarrie.

University of Wisconsin: Mr. Richard Francis De Mar, Mr. Robert Irving Jennrich, Mr. William John Kammerer, Mr. William Charles Lordan, Mr. Thomas La Rue McCoy, Mr. Clark Thompson Miller, Mr. Hiram Paley, and Mr. Raymond Warren Rishel.

The Secretary announced that the following had been admitted to the Society in accordance with reciprocity agreements with various mathematical organizations: Deutsche Mathematiker Vereinigung: Dr. Hans Konrad Schuff, Institut für Spektrochemie und angewandte Spektroskopie, Bau, Germany; Indian Mathematical Society: Dr. Alladi Ramakrishnan, University of Madras, Madras, India; Islenzka Staerơfraeöafelagiǒ: Professor Leifur Asgeirsson, University of Iceland; London Mathematical Society: Professor Reuben Louis Goodstein, University College of Leicester; Schweizerische Mathematische Gesellschaft: Dr. Henrich Guggenheimer, The Hebrew University, Jerusalem, Israel; Svenska Matematikersamfundet: Mr. Olof Edgar Asplund, Royal Institute of Technology, Stockholm, Sweden; Wiskundig Genootschap te Amsterdam: Professor Jacob Ridder, State University of Utrecht, Utrecht, Netherlands. 
The following appointments by the President were reported: to the joint committee on the Employment Register: Mr. George Patterson and Professor Ray Berkowitz. (Committee now consists of J. S. Frame, Chairman, H. M. Bacon, Ray Berkowitz, W. M. Hirsch, Morris Ostrofsky, George Patterson, and J. A. Ward); as a program committee for the Symposium in Applied Mathematics to be held in April 1956 in conjunction with the Society's Chicago meeting: William Prager, Chairman, L. M. Graves, Norman Levinson, Marston Morse, J. J. Stoker, and L. H. Thomas; as tellers for the 1955 election: Professors Arlen Brown, David Gale, and John Wermer; as a Committee to Recommend the Award of the Cole Prize in Number Theory: Professors Richard Brauer, Chairman, Leonard Carlitz, and Morgan Ward; as a Committee to Recommend to the Council Desirable Extensions of the Society's Activities: A. A. Albert, Wilfred Kaplan, J. L. Kelley, William Prager, and A. W. Tucker; as members of Committees to Select Hour Speakers (terms to expire December 31, 1957): Summer and Annual Meetings: G. P. Hochschild. (Committee now consists of E. G. Begle, Chairman, D. H. Lehmer, and G. P. Hochschild) ; Eastern Sectional Meetings: E. R. Kolchin. (Committee now consists of R. D. Schafer, Chairman, Lipman Bers, and E. R. Kolchin); Western Sectional Meetings: E. H. Spanier. (Committee now consists of J. W. T. Youngs, Chairman, P. V. Reichelderfer, and E. H. Spanier); Far Western Sectional Meetings: Arthur Erdélyi. (Committee now consists of V. L. Klee, Chairman, Ivan Niven, and Arthur Erdélyi); Southeastern Sectional Meetings: E. E. Floyd. (Committee now consists of J. H. Roberts, Chairman, G. B. Huff, and E. E. Floyd); as Chairman of the Committee on Places of Meetings for 1956: W. M. Whyburn. As a new member of this Committee: R. M. Thrall. (Committee now consists of W. M. Whyburn, Chairman, C. B. Morrey, and R. M. Thrall).

The following appointments to represent the Society were reported: at the 100th anniversary of the founding of the Polytechnic Institute of Brooklyn, October 8, 1955: Professor Samuel Borofsky; at the Centennial Homecoming Convocation of Albright College on October 22, 1955: Professor D. W. Western; at the inauguration of Paul S. Bachman as President of the University of Hawaii on November 9, 1955: Professor Christopher Gregory; at the inauguration of Howard Rothmann Bowen as the 7th President of the College at Grinnell College, Grinnell, Iowa, on November 13, 1955: Professor L. A. Knowler; and at the Academic Convocation held by Tufts University on the theme "The Role of the Small University in American Higher Education," on December 8, 1955: Professor C. T. Bumer. 
The Secretary reported that Professor S. S. Chern had accepted an invitation to deliver an hour address at the Annual Meeting of 1955 in Houston, Texas; that Dr. H. C. Wang had accepted an invitation to deliver an hour address at the April 1956 meeting in Monterey, California; and that Professor J. T. Schwartz had accepted an invitation to deliver an hour address at the April 1956 meeting at Columbia University.

The Council voted to grant permission to the Editors of Mathematical Tables and Other Aids to Computation to reproduce reviews from Mathematical Reviews.

The Council voted to request the Trustees to increase the total amount of subsidies to other journals by approximately $10 \%$ to allow for the increase in costs of publication.

The Bulletin Editorial Committee reported that 608 pages had been published in 1955, not including a supplement of 66 pages containing a ten-volume index. The Council voted to recommend to the Board of Trustees that the Bulletin be authorized to print 625 pages in 1956.

The Transactions and Memoirs Editorial Committee reported that the interval between receipt of a paper and publication has been reduced to about eight months. This reduction is largely due to a third volume of the Transactions published in 1955. The Council voted to recommend to the Board of Trustees that three volumes of 550 pages each be published in the Transactions during 1956.

The Proceedings Editorial Committee reported that the interval between the receipt of a manuscript and publication was approximately ten or eleven months. The Council voted to recommend to the Board of Trustees that 1006 pages be authorized for the 1956 Proceedings. Professors Raoul Bott, Paul Civin, Edwin Hewitt, and R. M. Thrall were reported as new Associate Editors of the Proceedings.

The Mathematical Reviews Editorial Committee reported that 1190 pages, not including the index number, would be published in volume 16.

The Council voted to accept an invitation from the Pennsylvania State University to hold the 1957 Summer Meeting at University Park, Pennsylvania. The Council voted to hold the Annual Meeting for 1957 in January 1958, as an experiment.

The Council approved the following times and places of sectional meetings: The University of Chicago, April 12-14, 1956; Massachusetts Institute of Technology, October 27, 1956; California Institute of Technology, November 17, 1956; University of Kentucky, Novem- 
ber 1956; University of Missouri, November 1957; and Wayne University, November 1959.

The Council voted to approve in principle a plan whereby any member of the Society may receive either the Transactions or Mathematical Reviews instead of the Proceedings on payment of a premium which will be a function of the member's subscription rates to these journals.

On the grounds that such liaison was no longer needed, the Council voted to eliminate the position of liaison officer to the Quarterly of Applied Mathematics.

The Council voted to co-sponsor a joint meeting of the Western Section of the Operations Research Society of America and the Southern California Chapter of the Institute of Management Sciences to be held at the University of California at Los Angeles, May 30-31, 1956.

The Council voted to request the Trustees to provide a subvention of $\$ 1000$ to the Institute of Mathematics of the National University of Mexico for support of a Symposium in Algebraic Topology to be held in the summer of 1956.

The Annual Business Meeting of the Society was held on Thursday, December 29, 1955. The Secretary reported that at this time the ordinary membership of the Society is now 4892, including 468 nominees of institutional members and 33 life members. The membership of the Society continues to show a substantial annual increase. There are also 133 institutional members. The total attendance at all meetings in 1955 was 2213; the number of papers read was 760; there were 13 hour addresses; one Gibbs Lecture; one Retiring Presidential address; and 9 papers at the Applied Mathematics Symposium. The number of members attending at least one meeting was 1525.

At the annual election, in which over 1300 votes were cast, the following officers were elected:

President Elect, Professor Richard Brauer.

Vice Presidents, Professor A. A. Albert and Nathan Jacobson.

Associate Secretaries, Professors V. L. Klee, Jr., J. H. Roberts, and J. W. T. Youngs.

Members of the Editorial Committee of the Bulletin, Professor G. B. Price for a term of two years and Professor J. C. Oxtoby for a term of three years.

Members of the Editorial Committee of the Proceedings, Professors R. P. Boas, Jr. and S. S. Chern.

Members of the Editorial Committee of the Transactions and Memoirs, 
Professors A. H. Clifford and Mark Kac.

Member of the Editorial Committee of the Colloquium Publications, Professor Deane Montgomery.

Member of the Editorial Committee of the Mathematical Reviews, Professor W. S. Massey.

Member of the Editorial Committee of the Mathematical Surveys, Professor I. J. Schoenberg.

Member of the Committee on Printing and Publishing, Professor A. W. Tucker.

Members of the Board of Trustees, Professor H. F. Bohnenblust for a term of two years and Professor W. T. Martin for a term of five years.

Representative on the Board of Editors of the American Journal of Mathematics, Professor Andre Weil.

Members-at-large of the Council, Professors Einar Hille, J. L. Kelley, Hans Samelson, A. H. Taub, and A. D. Wallace.

Professor R. H. Cameron urged a return to the former practice of printing abstracts in the next issue of the Bulletin after receipt rather than with the report of the meeting at which the paper was presented. There was a lively discussion of this and of other plans for making the abstracts more useful to members of the Society. The Secretary agreed to have a careful study made of these plans.

Abstracts of the papers presented follow. Those having the letter "t" after their numbers were read by title. Where a paper has more than one author, the paper was presented by that author whose name is followed by "(p)." Mr. L. W. Anderson was introduced by Professor A. D. Wallace, Mr. Baker by Professor H. A. Arnold, Dr. Krickeberg by Professor J. L. Doob, and Dr. Thorne by Dr. J. H. Curtiss.

\section{Algebra And Theory of Numbers}

163. W. W. Boone: The equivalence of the word problem and Magnus' extended word problem.

An arbitrary finitely presented group $\&$ can be extended to a finitely presented group '\$S' such that a solution of the word problem for $\$)^{\prime}$ implies the solution of the extended word problem (Magnus, Math. Ann. vol. 106, p. 297) for (3. Let \&5 have generators $g_{1}, g_{2}, \cdots, g_{n}$ and relations $R_{1}, R_{2}, \cdots, R_{s}$; let $m$ be an integer such that $0 \leqq m \leqq n$. We define (S)' to be the group with generators $g_{1}, g_{2}, \cdots, g_{n}, k$ and relations $R_{1}, R_{2}, \cdots, R_{s}, k g_{i}=g_{i} k$ for $1 \leqq i \leqq m$. Let $\Sigma$ (Let $\Gamma$ ) be a variable for words made up of $g_{1}, g_{2}, \cdots, g_{n}$ (of $g_{1}, g_{2}, \cdots, g_{m}$ ). THEOREM: There is a $\Gamma$ such that $\Sigma=\Gamma$ in (\$) if and only if $k \Sigma=\Sigma k$ in $\mathfrak{S}^{\prime}$. This theorem should be compared with the unsolvability result, Part I, p. 232. (References are to the parts of Certain simple unsolvable problems of group theory, Proc. K. Akad. Wetensch. A, Parts I and II, vol. 57; Part III, vol. 58; Part IV, vol. 58.) A proof, in (S)', that $k \Sigma=\Sigma k$ can be altered (Part I, Section 3 and Part II, p. 493, line 14-but $k$ here is treated like $q$ there) as follows: (1) Eliminate 
all uses of $k^{-1}$. (The methods of Part II, Lemma 7 and Theorem III, Case 1, suffice but are unnecessarily complicated.) (2) Eliminate all replacements of $g_{i}^{+1} k$ by $k_{i}^{+1}$ and $g_{i}^{-1} k$ and $k g_{i}^{-1}$. (Amend the marker convention so as to regard $k g_{i}^{-1}=g_{i}^{-1} k$ as an independent relation. Then for $\cdots, B g_{i}^{ \pm 1} k C, B k g_{i}^{ \pm 1} C, \cdots$ use $\cdots, B g_{i}^{ \pm 1} k C$, $B g_{i}^{ \pm 1} k g_{i}^{\mp 1} g_{i}^{ \pm 1} C, B g_{i}^{ \pm 1} g_{i}^{\mp 1} k g_{i}^{ \pm 1} C, B k g_{i}^{ \pm 1} C, \cdots$ ) (3) Interchange operations until $U$, the subsequence of operations performed on $k$ or right of $k$ precedes the operations left of $k$. The word resulting from the last operation of $U$ is of form $\Gamma k$. (Received December 30, 1955.)

\section{4t. J. L. Brenner: Some free groups of matrices.}

In Doklady Akad. Nauk SSSR (N.S.) vol. 57 (1947) pp. 657-659 [Math. Reviews vol. 9 (1948) p. 224], Sanov proved that the matrices $I+2 e_{12}, I+2 e_{21}$ generate a free group, which consists of all $\left(a_{i j}\right)_{0<i, j<3}$ such that $\operatorname{det}\left(a_{i j}\right)=1, a_{11} \equiv a_{22} \equiv 1(\bmod 4)$; $a_{12} \equiv a_{21} \equiv 0(\bmod 2)$. The generalization obtained is as follows. If $m \geqq 2$, the matrices $I+m e_{12}, I+m e_{21}$ generate a free group, which consists of all $\left(a_{i j}\right)$ such that det $\left(a_{i j}\right)=1$, $a_{11} \equiv a_{22} \equiv 1\left(\bmod m^{2}\right), a_{12} \equiv a_{21} \equiv 0(\bmod m)$, and the ratio $\left|a_{11} / a_{12}\right|$ is not strictly between $m / 2+\left(m^{2}-4\right)^{1 / 2} / 2$ and $m / 2-\left(m^{2}-4\right)^{1 / 2} / 2 ; m$ need not be an integer. (Received November 4,1955 .)

165. J. L. Brenner: The linear homogeneous group. III. Preliminary report.

Let $R$ be the ring of rational integers. The normal and characteristic subgroups of the general linear group $G L_{n}[R]$ are determined $(n>2)$. The method is extended to the case in which the underlying ring is euclidean. It is proved that every subgroup of $G L_{n}[R]$ of finite index contains a congruence subgroup, verifying a conjecture communicated by M. Newman. Some miscellaneous results and examples are given for the case $n=2$. This article will be published in the Annals of Mathematics. Research sponsored by Office of Ordnance Research, U. S. Army. (Received November 4, 1955.)

166. A. H. Copeland ( $\mathrm{p}$ ) and Frank Harary: Periodic sets of ordinals.

A subset $A$ of the positive integers is said to have period $n$ provided $T_{\beta n}(\alpha)=\alpha+\beta n$ is in $A$ if and only if $\alpha$ is in $A$ for every positive integer $\beta$. We extend this definition to sets $A$ of transfinite ordinals by replacing the usual sum by the natural sum and allowing $\alpha$ and $\beta$ to range over all values preceding some ordinal of the form $\omega^{\mu}$. In the case of the integers it is sufficient to restrict $\beta$ to be 1 . Associated with every order type $\omega^{\mu}$ and every integer $n \geqq 2$ there is a minimal set $F$ (called a free set) such that any set with period $n$ is a union of translations $T_{\beta n}$ of some subset of $F$. The representation space of an arbitrary boolean algebra can be regarded as a free set $F$ of a properly chosen order type. The representation of the boolean algebra as subsets of $F$ induces a representation as subset of $\omega^{\mu}$ with period $n$. By adding sets with various periods, one can extend this boolean algebra in such a manner that it becomes implicative. (Received November 14, 1955.)

\section{7t. R. H. Crowell: Forests and determinants.}

Various forms of an important combinatorial identity relating determinants to maximal trees of a connected graph exist in the literature. The purpose of this paper is: (i) To present a generalization, called the matrix-forest theorem, of previous results, in which forests replace trees and the corresponding extension is to arbitrary 
principal minors. (ii) To give a precise proof exhibiting the result as a formal identity of ring addition and multiplication. Let $V$ be the set of $n$ vertices, and $E$ the set of edges, of an arbitrary, oriented (each edge has an initial point and a terminal point) graph $\$ 3$. Where $\Re$ is an associative, commutative ring with a nonzero multiplicative identity, form $\Re(E)$ equal to the ring of polynomial forms over $\Re$ in the edges $E$. Define $\phi: V \times V \rightarrow \Re(E)$ by: for any $u, v \in V$, if $u \neq v$, then $\phi(u, v)=$ the sum of the edges in $E$ whose initial point is $u$ and terminal point is $v, \phi(u, u)=-\sum_{w \in V-u} \phi(w, u)$. The set $\phi(V \times V)$ is "the matrix" of the theorem, and for any subset $R \subset V$ of cardinality $r$, the corresponding principal minor $\Delta$ of order $n-r$ is easily defined: $\Delta=\sum_{\pi \in \mathfrak{S}^{n-r} \epsilon_{\boldsymbol{\pi}}}$ $\prod_{u \in V-R} \phi(u, \pi u) . \widetilde{S}^{n-r}$ is the group of permutations $\pi$ of $V-R$ and $\epsilon_{\pi}$ is the parity of $\pi$. A maximal rooted forest $F$ of \& with roots $=R$ is any acyclic subgraph of \& such that $V C F$, no vertex is the terminal point of more than one edge of $F$, and the set $R$ constitutes precisely those vertices which are the terminal points of no edge of $F$. The set of all such rooted forests is denoted $\mathfrak{F}_{R}$ and the form $\wp \in \Re(E)$ is defined: $\wp=\sum_{F \in \mathfrak{F}_{R}} \prod\{e: e \in F \cap E\}$. The matrix-forest theorem is the identity $\wp=(-1)^{n-r} \Delta$. The proof is based on the proof of the result for $r=1$ given in (R. Bott and J. P. Mayberry, Matrices and trees, "Economic Activity Analysis," O. Morgenstern, Ed., New York, Wiley, 1954). (Received October 24, 1955.)

\section{8t. W. E. Deskins: On bound algebras.}

A linear associative algebra $A$ with radical $N$ is right bound if all elements of $A$ which annihilate $N$ on the right are contained in $N . A$ is strictly right bound if all of its homomorphic images are right bound. Theorem. An algebra $A$ is expressible as a (vector space) direct sum of left ideals, $A_{0}+A_{1}+\cdots+A_{n}$, where $A_{0}$ is semisimple and $A_{i}, i=1, \cdots, n$, is strictly right bound. Theorem. If $A$ is strictly right bound, if the radical $N$ of $A$ is of index $r$, and if $N-N^{r-1}$ is of order $n_{r}$, then the order of $\bar{A}=A-N$ is at most $n_{r}^{2}$. These theorems and their duals, applied to the bound algebras of M. Hall (Trans. Amer. Math. Soc. vol. 48 (1940) pp. 391-404), yield new results concerning the relative sizes of an algebra and its radical. (Received November 14, 1955.)

\section{9t. Walter Feit: On the modular characters of finite groups.}

Let $G$ be a finite group of order $g$, and let $K$ be an algebraic number field with the property that every absolutely irreducible representation of $G$ can be written with coefficients in $K$. Let $p_{1}, \cdots, \mathfrak{p}_{s}$ be prime ideals in $K$ which divide distinct rational primes $p_{1}, \cdots, p_{s}$, let $a=p_{1}, \cdots, p_{s}, a=p_{1}, \cdots p_{s}$. Two matrices $A$ and $B$ whose coefficients are local integers with respect to each $\mathfrak{p}_{i}$ are said to be $\mathfrak{a}$-similar if there exists a unimodular matrix $P$ with such coefficients, such that $A=P B P^{-1}$, an element of $G$ is said to be $a$-regular if its order is relatively prime to $a$. The regular representation of $G$ is $\mathfrak{a}$-similar to a direct sum $\mu_{1} \oplus \cdots \oplus \mu_{s} \bmod \mathfrak{a}$ such that no $\mu_{i}$ is $\mathfrak{a}$-similar to a direct sum mod $a$. We define the character $\Phi_{i}$ of each $\mu_{i}$ as it is done for representations mod p (see Brauer and Nesbitt, Ann. of Math. vol. 42 (1941) pp. 556-590) so that $\Phi_{i}(x)$ is an algebraic number in $K$ for every $a$-regular element $x$ of $G$. In analogy with the results in the above mentioned paper we show, (1) $\Phi_{i}$ is an ordinary character which vanishes for all elements not $a$-regular, (2) The number of non a-similar representations $\mu_{i}$ mod $a$ equals the number of classes of $a$-regular elements of $G$, and the corresponding $\Phi_{i}$ are linearly independent. Cartan invariants $c_{i j}$ are defined by $g c_{i j}$ $=\sum \Phi_{i}(x) \overline{\Phi_{i}(x)}$ where the sum ranges over all $x$ in $G$. These can then be used to generalize a result of Brauer and Nesbitt as follows. If $g=g^{\prime} p^{b} g^{c}$, where $p$ and $q$ are primes which do not divide $g^{\prime}$, and if there are exactly $p^{b} g^{c}$ elements in $G$ whose order 
divides $p^{b} g^{c}$, then these elements form a normal subgroup of $G$. (Received November 7, 1955.)

\section{0t. Casper Goffman: Concerning lattice homomorphisms.}

It is shown that if $L$ is a distributive lattice with a minimal element then, for every ideal $J C L$, there is one and only one homomorphism of $L$ onto a disjunctive lattice for which $J$ is the kernel. This is the maximal homomorphism whose kernel is $J$. For the case where $J=[0]$, the residue classes are the "fillets" in $L$. For the case where $L$ is relatively complemented, the known uniqueness theorem is obtained as a corollary. (Received August 19, 1955.)

\section{Arno Jaeger: On differentiations in commutative rings of square- free nonzero characteristic.}

Let $R$ be a commutative ring with identity 1 of characteristic $p q>0$ ( $p$ and $q$ two distinct prime numbers) in which each nonzero divisor has an inverse. A countable family of mappings $D=\left(D^{i}\right)_{i=0,1,2} \ldots$ of $R$ into itself is called a differentiation if $D^{0} x=x, D^{i}(x+y)=D^{i} x+D^{i} y, D^{i}(x y)=\sum_{\alpha+\beta=i} D^{\alpha} x D^{\beta} y D^{i} D^{i} x=C_{i+j, i} D^{i+j} x$ holds for all $x, y \in R, i=0,1,2, \cdots$, and, in particular, a differentiation with respect to $u(\in R)$ if $D^{1} u=1$ holds. An element $v$ of $R$ is called $D$-regular if $D v$ is a nonzero diviser and if there exists such a $v$ in $R$ then $D$ is called regular. If $D$ is regular and $x$ is $D$ regular there exists a differentiation $\Delta$ with respect to $x$ such that $D$ can be derived from $\Delta$ by means of the chain rule. An element $w$ of $R$ is $D$-integrable in $R$ if and only if $p D^{q-1} w=q D^{p-1} w=0$ holds; and if the integral exists a particular integral can be constructed by the use of operators involving differentiations with respect to elements. These theorems are the foundation of a general theory of differentiations in commutative rings of any squarefree nonzero characteristic. (Received November 14, 1955.)

172. B. W. Jones (p) and G. L. Watson: Indefinite ternary quadratic forms.

Using concepts closely related to the spinor genera of Eichler, the authors define a multiplicative group $\Gamma_{d}$ of square-free integers prime to $d$, the determinant of the indefinite ternary quadratic form $f$. Further, they show that $\Gamma_{d}$ has a subgroup $\gamma(f)$ consisting of all those elements of $\Gamma_{d}$ which are denominators of rational automorphs of $f$, where by the denominator of a matrix is meant the least common multiple of the denominators of its elements. They show that the number of classes in the genus of $f$ is equal to the order of the factor group $\Gamma_{d} / \gamma(f)$. Also they show that if an integer $n$ is represented by at least one but not by all of the classes of forms in the genus of $f$ and if $d n=n_{1} n_{2}^{2}$, where $n_{1}$ is square-free, then $1 . n_{1}>1 ; n_{1}$ divides $d$; if $d$ is odd, $n_{1} \equiv 1$ (mod 8). 2. If $p$ is a prime not dividing $2 d$ and $\left(n_{1} \mid p\right)=-1$, then $p$ cannot divide $n_{2}$. 3 . The number of classes in the genus which represent $n$ is equal to the number of classes that do not. (Received November 10, 1955.)

\section{3t. Tosio Kato and Olga Taussky: Normal matrices.}

It is shown that a finite matrix $A$ is necessarily normal if $A\left(A A^{*}-A^{*} A\right)=\left(A A^{*}\right.$ $\left.-A^{*} A\right) A$. The elements of $A$ are either complex numbers or taken from a formally real field, or from a field with an involution $a \rightarrow \bar{a}$ where $\sum a_{i} \bar{a}_{i}=0$ implies $a_{i}=0$. The same property also holds for bounded operators in the Hilbert space. It can further be shown that $\left(A,\left(A, *\left(A,\left(A^{*}, \cdots,\left(A, A^{*}\right) \cdots\right)=0\right.\right.\right.$ implies $\left(A, A^{*}\right)=0$ where $(X, Y)=(X Y-Y X)$. If the finite matrix is a $2 \times 2$ matrix then $\left(A, A, A, A^{*}\right)$ 
$=k\left(A, A^{*}\right)$ where $k$ is a constant which vanishes if $A$ has a double characteristic root. (Received October 20, 1955.)

\section{4t. J. H. B. Kemperman: On complexes in a group.}

Let $A=\{a\}$ and $B=\{b\}$ be finite subsets of an arbitrary group and let $C=A B$ be the set of all the elements $c=a b$. Let $k=[A]+[B]-[A B]$, where $[D]$ denotes the number of elements in the set $D$. Theorem. Each element $c_{0}$ in $C$ admits at least $k$ different representations of the form $c_{0}=a b$. This verifies the conjecture of the author's paper: Complexes in arbitrary groups, Bull. Amer. Math. Soc. Abstract 61-6-655. (Received November 14, 1955.)

\section{Naoki Kimura: On finite monothetic semigroups.}

A semigroup $M$ will be called monothetic, if $M$ is generated by one element. Let $M$ be a finite monothetic semigroup, then $M$ is characterized up to isomorphism by two positive integers one of which is called a length and the other a period. A semigroup whose length is $l$ and whose period is $p$ will be called of type $C(l, p)$. All types $C(l, p)$ where $l, p$ run over all positive integers form a complete lattice isomorphic with direct product of countable number of chains of positive integers by defining a suitable order. All homomorphic images and all subsemigroups of a semigroup of type $C(l, p)$ are determined. The lattice of all subsemigroups of a semigroup of type $C(l, p)$ ordering by set-inclusion is distributive if $l \leqq 5$, but is not even modular if $l \geqq 6$. Further a notion of tensor product is introduced and discussed on application to some decomposition theorem of semigroups. (Received November 16, 1955.)

176t. Walter Ledermann and B. H. Neumann: On the order of the automorphism group of a finite group. II.

A function $g=g(h, p)$ of the positive integer $h$ and the prime number $p$ is found with the property that every finite group whose order is divisible by $p^{g}$ has an automorphism group whose order is divisible by $p^{h}$. The smallest such $g$ satisfies $g(1, p)=2$ and $g(h, p) \leqq(h-1)^{3} p^{h-1}+h$ for $h \geqq 2$. Results for small values of $h$, or for special classes of groups, had previously been obtained by J. E. Adney, Jr. [Bull. Amer. Math. Soc. Abstract 61-2-166 (1955)]; I. N. Herstein and J. E. Adney [Amer. Math. Monthly vol. 59 (1952) p. 309]; E. Schenkman [Proc. Amer. Math. Soc. vol. 6 (1955) p. 6]; W. R. Scott [Proc. Amer. Math. Soc. vol. 5 (1954) p. 23]. The method is a refinement of that used in the first part [Proc. Roy. Soc. London (A), in print, cf. Bull. Amer. Math. Soc. Abstract 61-6-657] and employs the Schur multiplicator and its properties; cf. J. Schur [J. Reine Angew. Math. vol. 127 (1904) p. 20 and vol. 132 (1907) p. 85]. (Received November 14, 1955.)

\section{Mark Lotkin: The diagonalization of skew-hermitian matrices.}

Hermitian matrices may be diagonalized by an infinite sequence of unitary transformations. Each of these transformations may be chosen in such a manner as to annihilate a pair of selected off-diagonal elements. In the diagonalized matrix, the elements represent the characteristic values of the hermitian matrix. This procedure, originally due to Jacobi, has been extended to skew-hermitian matrices. The rate of convergence is the same as that for the hermitian case. (Received October 28, 1955.)

178. Ivan Niven: $A$ single rational approximation to a complex number. 
Let $R(i)$ denote the field obtained by adjoining $i$ to the rational numbers. It is proved that given any complex number $\theta$ there exists at least one pair $p, q \neq 0$ of integers of $R(i)$ such that $|\theta-p / q| \leqq\left(2-3^{1 / 2}\right)^{1 / 2}|q|^{-2}$. This result becomes false if $\left(2-3^{1 / 2}\right)^{1 / 2}$ is replaced by any smaller constant. A similar result for the approximation of any real number by a rational number was given by A. V. Prasad, J. London Math. Soc. vol. 23 (1948) pp. 169-171. (Received November 14, 1955.)

\section{R. H. Oehmke: Shrinkable algebras of level 2.}

Let $\mathfrak{P}$ be an algebra over the field $\mathfrak{F}$ of characteristic not 2,3 or 5 and $w$ an element of $\mathfrak{A}$. Use the symbols $R_{w}$ and $L_{w}$ to denote the operations of right multiplication and left multiplication by the element $w$, the symbol $T(w)$ to denote either of these multiplications, and the symbol $T(x, y, z)$ to denote a multiplication $T(w)$ defined for a product $w=x y z$ with an unspecified association. Then an algebra is said to be shrinkable of level 2 if every $T(x, y, z)$ is identically equal to a finite linear combination of products $T_{1}\left(w_{1}, w_{2}\right) T_{2}\left(w_{3}\right), T_{1}\left(w_{1}\right) T_{2}\left(w_{2}, w_{3}\right)$, and $T_{1}\left(w_{1}\right) T\left(w_{2}\right) T_{3}\left(w_{3}\right)$ over the field $\mathfrak{F}$ where $w_{i}, w_{j}$ and $w_{k}$ represent the three factors $x, y$ and $z$. Two of these algebras $\mathfrak{A}$ and $\mathscr{B}$ are said to be quasi-equivalent if the algebras are isomorphic as vector spaces and if the product $a \cdot b$ of $\mathfrak{B}$ can be expressed in terms of the product $a b$ of $\mathfrak{A}$ by $a \cdot b=\lambda a b+(1-\lambda) b a$ for an element of $\mathfrak{A}$ that is not equal to $1 / 2$. The class of noncommutative shrinkable algebras of level 2 with a unity element is divided into subclasses of quasi-equivalent algebras in order to facilitate the investigation for new simple power-associative algebras. (Received November 14,1955.)

\section{B. W. Volkmann: A theorem on the set of perfect numbers.}

Let $\sigma(n)$ denote the sum of all positive divisors of the integer $n$ and let $U_{i}$ be the set of odd numbers $n$ with $\sigma(n)=2^{i} n(i=1,2, \cdots)$. Then it is shown that the number of its elements not exceeding $x$ is $U_{i}(x)=O\left(x^{\alpha}\right)$ where $\alpha=1-(2(i+2))^{-1}$. Since $U_{1}$ is the set of odd perfect numbers, the case $i=1$ has, by virtue of known properties of the even perfect numbers, the immediate corollary that the set $V$ of all perfect numbers satisfies the condition $V(x)=O\left(x^{5 / 6}\right)$. The proof is based on a lemma due to $\mathrm{H}$. J. Kanold (J. Reine Angew. Math. vol. 194 (1955) pp. 218-220) stating that any perfect or multiply perfect number has a factor $k^{2}$ such $k^{2} \geqq(p+1) / 2, p$ being the largest and prime factor of $n$. Full proofs are given in a paper forthcoming in the J. Reine Angew. Math. (Received November 16, 1955.)

181. D. W. Wall: Characterizations of uniserial and generalized uniserial algebras in terms of Frobenius type algebras.

Let $A$ be an algebra over an algebraically closed field $K$. It is known (Nakayama, Ann. of Math. vol. 42 (1941) pp. 1-21) that the following are equivalent: (1) $A$ is a uniserial algebra. (2) For every two-sided ideal $Z, A / Z$ is a Frobenius algebra. (3) For every two-sided ideal $Z, A / Z$ is a weakly symmetric algebra. The purpose of this paper is to give an extension of this result (Theorem 2) and a parallel result for generalized uniserial algebras (Theorem 1). Theorem 1. $A$ is a generalized uniserial algebra if and only if for every two-sided ideal $Z, A / Z$ is a QF-2. An algebra is QF-2 if every primitive left and right ideal has a unique minimal subideal (Thrall, Trans. Amer. Math. Soc. vol. 64, pp. 173-183). By assuming this property for every $A / Z$, it is possible to construct a unique composition series for each primitive ideal and thus prove that $A$ is generalized uniserial. The converse follows easily from the known properties of the algebras. Theorem 2. $A$ is uniserial if and only if for every two-sided ideal $Z, A / Z$ is quasi-Frobenius. Since every quasi-Frobenius algebra is $Q F-2$, Theo- 
rem 1 implies that if every $A / Z$ is quasi-Frobenius then $A$ is generalized uniserial. It remains to show that $A$ is the direct sum of two-sided ideals which are themselves primary algebras. This is done by using the dualities that exist between primitive left and right ideals in every $A / Z$. The converse follows from the earlier result stated above. (Received November 9, 1955.)

182. G. P. Weeg (p) and Bernard Vinograde: The defect of completely primary algebras. Preliminary report.

Let $A=\operatorname{Ext}(R, g, N)$ be an associative extension of the finite-dimensional $R-R$ module $N$ by the division algebra $R$ with $g$ a noncobounding 2-cocycle in $C^{2}(R, N)$ such that $N=R g(R, R) R$ is the radical of $A$. If for some left basis $n_{1}, \cdots, n_{d}$ of $N$ it is true that $g(x, y)=\sum p_{i}(x, y) n_{i}$ for all $x, y$ in $R$, where $p_{i}(x, y)$ is in $R$, then we call the $p_{i}$ 's projections of $g$ with respect to the $n_{i}$ 's. It is shown that: (1) $N$ is in the center of $A$ if and only if every possible projection of $g$ is a cocycle; (2) When $N$ is in the center of $A$ then all the projections of $g$ are cobounding if and only if $g$ is cobounding; (3) When $A$ is considered modulo $N^{2}$, then (a) $A$ is generated by every residue system modulo $N^{2}$ (that is, $A$ is maximally uncleft) if and only if all projections of $g$ are noncobounding, (b) to every set of linearly independent noncobounding cocycles there exists a maximally uncleft extension $A$ by $R$. Applications are made to pure inseparable $R$ 's. (Received November 9, 1955.)

\section{ANALYSIS}

183t. Shmuel Agmon: The Dirichlet problem for linear elliptic partial differential equations with constant coefficients and of arbitrary order.

An approach to the existence theory is outlined which can be considered as the generalization to higher order equations of the classical procedure of representing the solution as a multiple layer potential. The fundamental solution is not used directly for this purpose. Instead, inspired by a treatment of Pleijel of the biharmonic equation (Proceed. Symp. Spectral Theory and Differential Problems, Stillwater 1951, pp. 413-437), the Poisson kernels obtained from the explicit solution of the Dirichlet problem for the half-space are employed. One first tries to get the desired solution as an average on the boundary of the domain of these Poisson kernels. Introducing the Banach space of $m$-vectors: $x=\left(\phi_{0}(Q), \cdots, \phi_{m-1}(Q)\right)$, where $2 m$ is the order of the equation and $\phi_{k}(Q)$ are functions belonging to $C_{m-1-k+\alpha}(0<\alpha<1)$ on the sufficiently smooth boundary, one first reduces the problem to solving: $\left(^{*}\right) X+T(x)$ $=y$ where $T$ is a completely continuous operator and $y$ is the vector of the prescribed boundary values. Thus the problem is certainly solvable in this way when $x+T(x)=0$ admits no nontrivial solutions. Furthermore, even if nontrivial solutions of the homogeneous equation exist, one can always obtain the solution of the Dirichlet problem as a multiple layer potential of the above form and a linear combination of some fundamental solutions with poles outside the domain. (Received November 14,1955.)

184. Nachman Aronszajn: A unique continuation theorem for elliptic partial differential equations and inequalities of second order.

The unique continuation is proved for solutions $u$ of partial differential inequalities of the type $|A u| \leqq c \sum\left|\partial u / \partial x^{k}\right|$ for $|x|<R$ where $c>0$ is a constant and $A$ is elliptic of second order with variable coefficients and principal part $\sum a^{i j} \partial^{2} u / \partial x^{i} \partial x^{i}$. The proof is obtained by extending to the present case an inequality proved by $\mathrm{E}$. Heinz 
(Nachr. Ges. Wiss. Göttingen (1955) pp. 1-12) for the case $A=\Delta$, the ordinary laplacian. To obtain this extension one considers first the riemannian metric $\sum a_{i j} d x^{i} d x^{i}$, $\left\{a_{i j}\right\}$ being the inverse matrix of $\left\{a^{i j}\right\}$. Denoting by $r$ the geodesic distance from the origin to a variable point $x$, a suitably large constant $\alpha>0$ can be chosen such that by putting $\phi(r)=\exp \left(-\alpha r^{2}\right)$ and $A^{\prime}=$ the Beltrami-Laplace (B.-L.) operator corresponding to the riemannian metric $d s^{2}=\phi(r) \sum a_{i j} d x^{i} d x^{i}$, Heinz's inequality can be proved when put in invariant form relative to $d s^{2}$ (i.e. ordinary laplacian replaced by $A^{\prime}$, distances, spheres, etc. replaced by geodesic distances, spheres, etc.). The proof of the inequality relies on the special form of the B.-L. operator in terms of geodesic polar coordinates, and proceeds by partial integrations in the main integral of the inequality. The role of the constant $\alpha$ is to render positive a crucial term in the final form of this integral. (Received November 14, 1955.)

185. J. H. Barrett: $A$ necessary condition for nonoscillation of $a$ system of second order differential equations. Preliminary report.

Consider the matrix second-order self-adjoint differential equation: (1) $\left(P(x) Y^{\prime}\right)^{\prime}$ $+Q(x) Y=0$, where, for $0 \leqq x<\infty, P(x)$ and $Q(x)$ are continuous real square matric functions and $P(x)$ is positive definite. Theorem: If the corresponding vector-matrix equation (2) $\left(P(x) \alpha^{\prime}\right)^{\prime}+Q(x) \alpha=0$ is nonoscillatory (i.e. has no pair of conjugate values for large $x$ ) then there exists a matrix solution $U(x)$ of (1) such that $\int^{\infty}[\operatorname{tr} P(x)]^{-1}$ - $\|U(x)\|^{-2} d x<\infty$. For the scalar case this is similar to a result of Wintner and Leighton (see Leighton, J. London Math. Soc. vol. 27 (1952) pp. 46-47) but the proof is simpler since it does not involve a polar transformation. Sufficient conditions for boundednes of all solutions of (1) (or (2)) have been given by G. Borg, R. Bellman, and the author. For any such set of conditions together with the assumption that $\int^{\infty}(\operatorname{tr} P)^{-1}=\infty$ it follows easily from the above theorem that the system (2) is oscillatory. Also, additional results along this line are obtained. (Received November 14, 1955.)

186t. R. W. Bass: A generalization of the functional relation $Y(t+s)$ $=Y(t) Y(s)$ to piecewise-linear difference-differential equations.

Let $A, B, P, Q$ be real constant $n \times n$ matrices, and $y, b, f$ real $n$-vectors, where $f$ is a step-function of the scalar $\Sigma$, and $b$ a constant. Let $\Sigma(t) \equiv b \cdot B y(t)$ (where $\cdot$ denotes scalar product) and $\Sigma_{1}(t) \equiv b \cdot B\left(P y(t)+Q f\left(\Sigma_{1}\left(t-t_{d}\right)\right)\right.$. Consider the system (*): $\dot{y}=A y+f\left(\Sigma_{1}\left(t-t_{d}\right)\right)$ during an interval $\left(t-2 t_{d}, t+2 t_{d}\right)$ for which it is known $a$ priori that $f$ is constant. If the general solution of $(*)$ for $y(0)=y_{0}$ is $y(t)=y(t) y_{0}$ $+Z(t) f$, then $y\left(t+t_{d}\right)=Y\left(t_{d}\right) y(t)+Z\left(t_{d}\right) f$. Corollary: Let $P=Y\left(t_{d}\right), Q=Z\left(t_{d}\right)$; then $\Sigma_{1}(t)=\Sigma\left(t+t_{d}\right)$, so that $(*)$ is in $\left(t, t+t_{d}\right)$ equivalent to $\dot{y}=A y+f(\Sigma(t))$. There are important applications to the design of control systems. (Received November 14, 1955.)

\section{R. W. Bass: On nonlinear repulsive forces.}

Consider the real system of second order differential equations $x^{\prime \prime}=f\left(x, x^{\prime}, t\right)$, where $x$ is an $n$-vector, and $f$ a continuous vector function (on the half-space $t \geqq 0$, $x_{i}, x_{i}^{\prime}$ unrestricted) satisfying $x \cdot f \geqq 0$ (· is scalar product); $|f|$ is subject only to a growth restriction $|f|\left\langle C_{K}\left|x^{\prime}\right|^{2}\right.$ (for $(t, x)$ in any compact set $K$ ). Then through each point $x_{0}$ there passes at least one unrestricted, bounded solution, and one (possibly restricted) unbounded solution. The bounded solutions $x(t)$ are "asymptotic" in the sense that the squared magnitude $r=|x(t)|^{2}$ is nonincreasing and convex from below; that is, $r \geqq 0, r^{\prime} \leqq 0$, and $r^{\prime \prime} \geqq 0$. The proof consists principally of $a$ priori majorisation of the "asymptotic" solutions and application of the Leray-Schauder fixed-point 
theory. The result generalizes theorems of Kneser on scalar differential equations, of Wintner on linear system (Amer. J. Math. vol. 68 (1946) pp. 173-178), and of Hartman and Wintner on linear systems. (Received November 14, 1955.)

188t. R. W. Bass: On the regular solutions at a point of singularity of a system of nonlinear differential equations.

This paper generalizes the Cauchy-Poincare existence theorem to singular systems of the form $z^{s i} w_{i}^{\prime}(z)=f_{i}\left(z, w_{1}, \cdots, w_{n}\right)(i=1, \cdots, n)$, where the $f_{i}$ are regular; $f_{i}(0,0, \cdots, 0)=0$; and $s_{i}$ are non-negative integers satisfying $0 \leqq s \equiv s_{1}+\cdots+s_{n}$ $<n$. It is asserted that there is a $k$-parameter family of solutions regular at $z=0$, where $n-s \leqq k \leqq n$; the solutions will have algebroid dependence on the $k$ parameters and any additional parameters in which the $f_{i}$ are given regular. This theorem generalizes results of Perron and Lettenmeyer on the linear case (cf. Bieberbach's recent book “...gewöhnliche Differentialgleichungen ...," pp. 161-172). The technique of the proof constitutes an application of the method of undetermined coefficients, combined with Wintner's fixed-point theorem for "analytic" mappings in a separable Hilbert space. Details will appear in the Amer. J. Math. (Received November 14, 1955.)

189. E. F. Beckenbach (p) and M. O. Reade: Generalized Laplacians and harmonic and subharmonic functions.

For harmonic, subharmonic, and convex functions the authors discuss characteristic properties which include previously discussed properties as special cases. In particular, the validity of a strengthened form of a conjecture of Osamu Ishikawa [Proc. Japan. Acad. vol. 30 (1954) pp. 686-690] concerning the operator $(1 / 2 \pi) \int_{0}^{2 \pi} u(x+r$ $\cdot \cos \theta, y+r \sin \theta) d \theta-\left(1 / \pi r^{2}\right) \int_{0}^{2 \pi} \int_{0}^{r} u(x+\rho \cos \theta, y+\rho \sin \theta) \rho d \rho d \theta$ is established. (Received November 14, 1955.)

190t. Garrett Birkhoff: Extensions of Jentzsch's Theorem. Preliminary report.

Let $C$ be any convex set of "positive" rays of an $(L)$-space into itself, and let $P$ be any linear transformation which carries $C$ into itself. If some iterated transform $C P^{r}$ of $C$ under $P$ has finite hyperbolic diameter relative to $C$, then, for any $f \in C$, the sequence of $f P^{n}$ converges geometrically to a unique characteristic ray $c \in C$. It is shown that this geometrical result implies various generalizations of Jentzsch's Theorem. (Received November 9, 1955.)

\section{Arlen Brown: Congruence of self-adjoint operators.}

Two self-adjoint operators $A$ and $B$ (on a Hilbert space) are said to be congruent if there exists a bounded operator $P$ with bounded inverse such that $B=P^{*} A P$. Using the congruence invariants obtained by Koethe in Math. Zeit. vol. 41 (1936) pp. 137152 (in a somewhat modified form) the following theorem is proved: If $A \geqq 0, B \geqq 0$ then $A$ and $B$ are congruent if and only if $A^{k}$ and $B^{k}$ are ( $k$ any positive number). It follows from this, according to a suggestion of Kaplansky's, that necessary and sufficient conditions that two closed operators $A$ and $B$ be equivalent are that $A^{*} A$ and $B^{*} B$ be congruent and that $A^{*}$ and $B^{*}$ have equal nullity. Thus the equivalence problem can be satisfactorily solved in terms of congruence invariants. An analogous theorem is proved concerning the similarity of operators $A$ and $B$ in the special case $A^{2}=B^{2}=0$. (Received November 14, 1955.)

192. H. D. Brunk: On an inequality for convex functions. 
Let $X(t)$ be given nondecreasing, continuous from the left, and bounded on $[a, b]$, and let $G(t)$ be a given function of bounded variation continuous from the right on $[a, b]$, with $G(a)=0, G(b)=1$. For an interval $I$, define $M(I)=\int_{1} G(t) d X(t) / \int_{1} d X(t)$, and define $G^{*}(t)=\inf _{v} \sup _{u} M(I)=\sup _{u} \inf _{v} M(I)$, where $u, v$ are respectively left and right end points of the interval $I$ containing $t$. The function $G^{*}$ can be shown to be the best nondecreasing approximant to $G$, in the sense of least squares, with respect to the function $X(t)$. It is shown that a necessary and sufficient condition that $\int_{[a, b]} f[X(t)] d G(t) \geqq f\left[\int_{[a, b]} X(t) d G(t)\right]$ for every continuous convex $f$ is that $G^{*}$ be a distribution function on $[a, b]$ ( $G^{*}$ is nondecreasing; it suffices then that it be bounded by 0 and 1 ). This theorem contains and is a consequence of the well-known theorem to the effect that the above inequality holds for every continuous convex $f$ if $G$ is a distribution function having total variation 1 . It contains also the inequalities discussed by E. M. Wright (An inequality for convex functions, Amer. Math. Monthly vol. 61 (1954) pp. 620-622). (Received November 14, 1955.)

193. H. D. Brunk, G. M. Ewing (p), and W. R. Utz: Minimizing integrals in certain classes of monotone functions.

Suppose given an interval $I=[a, b] ; \alpha(t), t=\left(t^{1}, \cdots, t^{n}\right)$, a suitable function measurable over $R_{n}$ with range in $I ; F(u, v)$, a suitable function on $I \times I$, such that, for fixed $u, F$ is strictly decreasing (increasing) for $a<v<u(u<v<b)$; a totally finite complete measure $\mu$ on a Borel field of subsets of $R_{n}$. Consider the integral over $R_{n}$, $J[\theta]=\int F[\alpha(t), \theta(t)] d \mu$, in the class $M$ of functions $\theta(t)$, each with range in $I$, monotone in each coordinate $t^{i}$, and such that $J$ exists finite. Also consider $J[\theta]$ in the subclass $M^{*}$ consisting of those $\theta(t)$ in $M$, each of which is a bivariate distribution function (or its negative) in each pair of variables $t^{i}, t^{j}$ when the others are fixed. The existence and uniqueness of minimizing functions in $M\left(M^{*}\right)$ are established under additional hypotheses on $F$ and representations are obtained for a minimizing function in $M$. Various results of Brunk, Ewing, and Reid (Bull. Amer. Math. Soc. Abstract 60-6684 ) are now extended to $n$ variables and more general integrals. (Received November $10,1955$.

\section{R. H. Cameron: Nonlinear Volterra functional equations and linear parabolic differential systems.}

In this paper certain nonlinear functional equations of the form $y(t)=x(t)+\Lambda(x \mid t)$ are studied from the point of view of determining conditions under which continuous solutions $x$ vanishing at $t=0$ exist for almost every $y$ of the same class. [Here "almost every" means all except a set of Wiener measure zero.] This question is shown to be closely connected with the question whether certain linear parabolic differential systems have minimal positive solutions. Both questions can be answered in terms of the values of certain Wiener integrals. (Received November 14, 1955.)

\section{5t. Paul Civin and Bertram Yood: Semi-reflexive spaces.}

Let $X$ be a Banach space and let $\pi$ be the canonical isomorphism of $X$ into its second conjugate space $X^{* *}$. Call $X$ semi-reflexive of order $n$ if $\pi(X)$ has finite deficiency $n$ in $X^{* *}$. An example of a semi-reflexive space of order one was given by R. C. James (Ann. of Math. vol. 52 (1950) pp. 518-527). Let $T$ be a continuous linear mapping of $X$ onto a Banach space $Y$ with kernel $E$. Then $X$ is semi-reflexive if and only if $Y$ and $E$ are semi-reflexive. Furthermore, if $X, Y, E$ are semi-reflexive of order $\alpha, \beta$ and $\gamma$ respectively, then $\beta+\gamma=\alpha$. Properties analogous to those for reflexive spaces are also established for semi-reflexive spaces. (Received November 14, 1955.) 
196t. Albert Edrei: Signs of the coefficients of real entire functions, bounded on the positive axis.

Let $f(z)=\sum a_{n} z^{n}$ be a real entire function, bounded for $z>0$. Let $\left\{\lambda_{v}\right\}$ denote the sequence, taken in its natural order, of all the subscripts $\lambda$ of those coefficients $a$ which determine a change of sign (for a precise definition, see G. P6lya and G. Szegö, $A u f$ gaben und Lehrsätze aus der Analysis, vol. 2, p. 37). Then $\sum \lambda_{v}^{-1}$ diverges. Conversely, if $\left\{\lambda_{v}\right\}$ is a given series of positive increasing integers and if $\sum \lambda_{v}^{-1}$ diverges, there exists a real entire function $f(z)$, bounded for $z>0$, and such that the coefficients $a$ of its expansion have the following properties: (i) $a_{n} \neq 0(n=0,1,2, \cdots)$; (ii) $a_{\lambda} a_{\lambda-1}<0$ if and only if $\lambda$ is a member of $\left\{\lambda_{v}\right\}$. (Received November 14,1955.)

\section{7t. Jacob Feldman: $A W^{*}$ embedded $A W^{*}$ algebras are weakly closed.}

Let $\mathcal{A}$ be an $A W^{*}$ algebra which is $A W^{*}$ embedded as operators on a Hilbert space, and let $\mathcal{B}$ be its weak closure. Then $\mathcal{A}=\mathcal{B}$. The proof proceeds as follows: when $\mathscr{B}$ possesses some separating normal state, one can use noncommutative integration theory, following a technique of Segal, to show that $A=\mathbb{B}$. The theorem then easily also follows when $B$ is a $C^{*}$ sum of such rings of operators. In the general case, the problem is first reduced to that of showing that $A$ contains the weak closure of any sequence of its elements. Let $A_{1}, A_{2}, \ldots$ be such a sequence; then the ring of operators $\mathcal{B}_{0}$ generated by this sequence has a countable dense subset. Let $\mathcal{C} A_{0}=A \cap \mathcal{B}_{0}$. Then $A_{0}$ is an $A W^{*}$ embedded $A W^{*}$ algebra with weak closure $B_{0}$. But it can be shown that if a ring of operators $\mathcal{C}$ has a weakly dense subset of cardinality $s$, then $C$ is a $C^{*}$ direct sum of rings of operators $\bigodot_{i}$ such that every set of orthogonal nonzero projections in one of the $\mathcal{C}_{i}$ has cardinality $\leqq \aleph$. Thus $\mathcal{B}_{0}$ is a $C^{*}$ sum of " $N$-finite" rings of operators. But an $\mathbb{N}_{0}$-finite ring of operators has a separating normal state, as shown by E. Griffin. Therefore $A_{0}=\mathscr{B}_{0}$, and $\mathcal{A}=\mathcal{B}$. (Received October 26, 1955.)

\section{L. R. Ford: Interval-additive propositions.}

A statement involving an interval will be called interval-additive if when true for each of two overlapping intervals it is true also for their union; for example, the statement that a function $f(x)$ is bounded in an interval, or continuous, or of constant sign, or strongly monotonic. The main theorem which is proved is that if an intervaladditive proposition is true at each point of a closed interval $I$ it is true for $I$. Here by truth at a point we mean truth in a subinterval of $I$ enclosing the point, with the convention that at an endpoint of $I$ the subinterval terminates at and includes the end point. Basic properties of a continuous function in a closed interval, or of sets of points, are easy applications of this theorem. (Received November 14, 1955.)

199. R. E. Fullerton: The construction of smoothed contours of a Fréchet surface.

Let $S$ be a Fréchet surface with representation $T$ defined over a disk $D$ in the plane and let $f$ be a Lipschitzian function defined on $E_{3}$. The contour defined by $f$ and $T$ for the real value $t$ is the boundary of the open set in $D$ for which $f(T(x))<t$. Cesari has defined smoothed contours by considering certain irreducible continua in the hyperspace of continua of constancy of $T$ in $D$. In this paper a method is given for constructing smoothed contours together with a proof that they are uniquely defined. It is shown that for a smoothed contour the topology in the hyperspace of continua of constancy is equivalent to the interval topology on certain equivalence classes of 
ends and prime ends corresponding to points of the contour. It is also proved that the generalized lengths of segments on the reduced contours are the same when computed from both sides of the contour and that if $S$ is nondegenerate, there exists a representation $T$ of $S$ for which all smoothed contours are arcs. (Received November 14, 1955.)

200. T. M. Gallie, Jr.: Region of convergence of Dirichlet series with complex exponents.

Hille (Ann. of Math. (1924)) has described a convex "maximal region" $D$ such that the Dirichlet series $\sum_{n=1}^{\infty} c_{n} \exp \left(-\lambda_{n} s\right)$, with complex exponents $\lambda_{n}$, does not converge outside of $D$ and converges absolutely in the interior of $D$ provided that $\lim (\log n) / \lambda_{n}=0$. This description was simplified somewhat by Valiron (Ann. Ecole Norm. (1929)). In the present paper it is observed that $D$ is simply the set of points $s$ for which $d(s)=\lim$ inf $\left[-\left|\lambda_{n}\right|^{-1} \log \left|c_{n} \exp \left(-\lambda_{n} s\right)\right|\right]$ is non-negative. It is proved for $s \in D$ that $d(s)$ is the distance from $s$ to the frontier of $D$ and that the series converges absolutely to an analytic function at every point of $D$ whose distance from the frontier is greater than $\lim \sup (\log n) /\left|\lambda_{n}\right|$. Thus the formula for $d(s)$ contains the Cauchy-Hadamard formula for Taylor series and a well known formula relating the abscissas of simple and absolute convergence for Dirichlet series with positive exponents. (Received November 7, 1955.)

201t. L. W. Green: Bounded solutions of Riccati systems. Preliminary report.

The matrix Riccati equation associated with a system of $n$ second order linear equations is investigated under (among others) the hypothesis of nonconjugacy. E. Hopf's results (Proc. Nat. Acad. Sci. U.S.A. vol. 34 (1948) pp. 47-51) in the case $n=1$ are extended, not without modifying his method, to show that there exists a matrix solution of the Riccati system with never-vanishing determinant whose associated (symmetric) quadratic form is bounded. As Hopf (loc. cit.) found, this yields an equation on the unit tangent bundle of any compact Riemannian manifold whose geodesics contain no conjugate points. Unfortunately, and contrary to the case of surfaces, the geometric significance of this last equation is still obscure. (Received November 14,1955 .)

202t. D. L. Guy: Weighted p-norms and Fourier transforms. Preliminary report.

The function $f(x) \in L^{\alpha, p}, 1<p<\infty,-1 / p<\alpha<1-1 / p$, if $\int f_{-\infty}^{\infty}|(x)| p|x|^{\alpha_{p}} d x<\infty$. Let $F(t)$ be the Fourier transform of $f(x)$ and $\Delta_{n}(x)=\int_{-\infty}^{\infty} \chi_{n}(t) F(t) e^{-i x t} d t$, where $\chi_{n}(t)$ is the characteristic function of $\left[2^{n-1}, 2^{n}\right]$ if $n \geqq 1$, of $[-1,1]$ if $n=0$, and of $\left[-2^{n}\right.$, $\left.-2^{n+1}\right]$ if $n \leqq-1$. The following transform analogue of a theorem of I. I. Hirschman [Memoirs of the American Mathematical Society, no. 15, p. 49] is proved by methods similar to those of Hirschman [op. cit. p. 29-51], Littlewood and Paley [Proc. London Math. Soc. vol. 42, p. 52] and Zygmund [Bull. Amer. Math. Soc. vol. 51, p. 439 and Fund. Math. vol. 30, p. 170]: if $f \in L^{\alpha, p}$, then there are constants $A_{1}$ and $A_{2}$ such that $A_{1} \leqq \int_{-\infty}^{\infty}\left(\sum_{n=-\infty}^{\infty}\left|\Delta_{n}(x)\right|^{2}\right)^{p / 2}|x|^{\alpha_{p}} d x / \int_{-\infty}^{\infty}|f(x)| p|x|^{\alpha_{p}} d x \leqq A_{2}$. By means of this result the transform analogue of the theorem of Marcinkiewicz [Studia Math. vol. 8, p. 78] is proved. (Received September 20, 1955.)

203. Guy Johnson, Jr.: Functions which have harmonic support. 
A real valued function $v(z)$ of the complex variable $z$ belongs to the class $(h s)$ in a domain $D$ if and only if for each $z_{0} \in D$ there exists a function $h(z)$ harmonic in $D$ such that $h(z) \leqq v(z)$ and $h\left(z_{0}\right)=v\left(z_{0}\right)$. These functions are continuous and subharmonic. A subclass of the negatives of these functions was used by Caratheodory (Amer. J. Math. (1937)) and Brelot observed (J. Math. Pures Appl. (1945) footnote p. 6) that there are subharmonic functions having continuous derivatives of order $p$ which are not in the class $(h s)$. If $\mathcal{F}$ denotes a subuniformly (i.e. uniformly on each compact subset of $D$ ) bounded family of functions $f(z)$ holomorphic in $D$, then $v(z)=\sup _{f}|f(z)|$ belongs to $(h s)$. If $D$ is simply connected, let ( $p l)$ denote the class of functions $v(z)$ such that $\log v(z) \in(h s)$, then $(p l) \subset(h s)$. Also $v(z) \in(p l)$ if and only if there exists a subuniformly bounded family $\mathcal{F}$ of functions $f(z)$ holomorphic and nonzero in $D$ such that $v(z)=\sup _{f}|f(z)|$. If $v(z) \in(h s)$ then there exists a sequence of functions of $(h s)$ having continuous derivatives of second order which converges subuniformly to $v(z)$ in $D$. A subuniformly bounded family $U$ of functions of the class $(h s)$ is normal. (Received November 14, 1955.)

\section{4t. Jacob Korevaar: Fourier transforms of generalized functions.}

A distribution $\phi$ is given by a fundamental sequence $\left\{f_{n}\right\}$, that is, a sequence of integrable functions such that for every finite interval $[a, b]$ there is a number $p$ and an associated sequence of $p$ times iterated indefinite integrals $\left\{f_{n}^{(-p)}\right\}$ which is uniformly convergent on $[a, b] . \phi$ is said to be of slow growth when one of its defining sequences $\left\{f_{n}\right\}$ is of slow growth: $\left|f_{n}^{(-r)}(t)\right| \leqq A(|t|+1)^{k},-\infty<t<\infty, n=1,2, \cdots$, for some associated sequence $\left\{f_{n}^{-r}\right\}$. When $\phi$ is of slow growth it may be given by a special sequence $\left\{g_{n}\right\}$ of slow growth such that $g_{n}(t)$ is continuous, equal to zero for $|t| \geqq n$, and of bounded total variation. Setting $\mathcal{F} g_{n}=(2 \pi)^{-1 / 2} \int_{-\infty}^{\infty} g_{n}(t) e^{-i u t} d t$ the sequence $\left\{\mathcal{F} g_{n}\right\}$ is fundamental and of slow growth. It defines a distribution $\psi$ of slow growth which may be called $\mathcal{F}_{\phi}$. Indeed, when $\left\{g_{n}^{*}\right\}$ is any other sequence of slow growth defining $\phi$ for which $\left\{\mathcal{F} g_{n}^{*}\right\}$ is fundamental, then the latter sequence defines the same $\psi$. In obvious notation, $\overline{\mathcal{F}} \psi$ also exists. It may be given by the fundamental

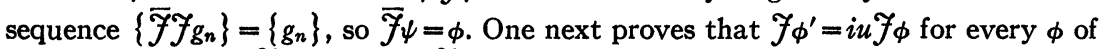
slow growth, that $\mathcal{F}_{1}=(2 \pi)^{1 / 2} \delta, \mathcal{F} \delta=(2 \pi)^{-1 / 2}$, etc. Thus the stage is set for various applications. (Received November 14, 1955.)

205. Stephen Kulik: Variations of a method for evaluating the zeros of analytic functions.

Let $f(z)$ and $\phi(z)$ be two analytic functions inside and on the circle $C$ of radius $r$; let $a$ be any zero of the function $f(z)$ of multiplicity $m$ inside the circle $C$, which may also be a zero of the function $\phi(z)$, but multiplicity not higher than $m-1$; the function $\phi(z)$ is otherwise arbitrary. Then it is shown that $a-z=\lim f(z) Q_{n-1}(z) / Q_{n}(z)$ and $a-z=\lim f(z) /\left(Q_{n}(z)\right)^{1 / n}, n \rightarrow \infty$, if $z$ is any number which is closer to $a$ than to any other zero of $f(z)$ or to the circle $C$, and $Q_{n}(z)$ is the determinant of $n$th order, the $k$ th line of which is $\mid \phi^{(k-1)}(z) /(k-1) !, f(z)^{(k-1)} /(k-1) !, f(z)^{(k-2)} /(k-2) !, \cdots, f^{\prime}(z)$, $f(z), 0, \cdots, 0 \mid$ and can be calculated by the recurrence formula $Q_{n}(z)=f^{\prime}(z) Q_{n-1}(z)$ $-f^{\prime \prime}(z) f(z) Q_{n-2}(z) / 2 !+\cdots+(-1)^{n-2} f^{(n-1)}(z) f(z)^{n-2} Q_{1} /(n-1) !+(-1)^{n-1} \phi^{(n-1)}(z)$ - $f^{n-1}(z) Q_{0} /(n-1)$ ! with $Q_{0}(z)=1, Q_{1}(z)=\phi(z), Q_{2}(z)=\phi(z) f^{\prime}(z)-\phi^{\prime}(z) f(z)$. Two particular cases are of special interest, $\phi(z)=1$ and $\phi(z)=f^{\prime}(z)$. If $f(z)$ is a polynomial with real coefficients and the roots of the equation $f(z)=0$ are all real, it is possible to obtain the upper and lower bounds for the roots, as close to each other as necessary, by using the two formulas above with a finite value of $n$ instead of $n \rightarrow \infty$ and $\phi(z)$ appropriately chosen. (Received November 7, 1955.) 
206. E. K. McLachlan: Extremal elements of a convex cone of subadditive functions.

Let $E$ be an arbitrary set of elements, and let $G$ be a class of subsets of $E$ such that the empty set and $E$ belong to $G$ and such that $X$ and $Y$ belong to $G$ implies $X \cup Y$ belongs to $G$. The set of non-negative, nondecreasing, and subadditive maps $f$ from $G$ into the real line form a convex cone $\mathcal{C}$. A set $X$ of $G$ is said to be $f$-equivalent to a set $Y$ of $G$ if for a given $f$ of $\mathcal{C}$ there exists a finite sequence of elements, $X=X_{0}$, $X_{1}, \cdots, X_{k}=Y$ of $G$ such that $X_{i} \subset X_{i+1}$ or $X_{i} \supset X_{i+1}$ and $f\left(X_{i}\right)=f\left(X_{i+1}\right)$ for $i=0,1, \cdots, k-1$. Let $C^{\prime}$ be the subcone of $\mathcal{C}$ which consists of those functions $f$ of $\mathcal{C}$ which have only a finite number of $f$-equivalence classes. Using a certain system of equations associated with each function $f$ of $\mathcal{C}^{\prime}$ a necessary and sufficient condition is given for $f$ to be an extremal element of $\mathcal{C}^{\prime}$. The terms convex cone and extremal element are used as they are defined in Bourbaki, XV. (Received November 14, 1955.)

207. G. R. MacLane: Meromorphic functions with specified asymptotic behavior.

Previously (Bull. Amer. Math. Soc. Abstract 60-2-252) the author gave an elementary proof of a sharpened version of Roth's theorem (Comment. Math. Helv. vol. 11). This theorem may be used to prove the following much stronger theorem. Let $\left\{D_{n}\right\}$ be an infinite sequence of domains in the z-plane such that: (1) the boundary of $D_{n}$ is a simple curve $\Gamma_{n}$ with both ends at $\infty$, (2) $\left(D_{m} \cup \Gamma_{m}\right) \bigcap\left(D_{n} \cup \Gamma_{n}\right)=0$ for $m \neq n,(3)\left(D_{n} \cup \Gamma_{n}\right) \cap(|z| \leqq R)=0$ for $n>n_{0}(R)$, and (4) $\left(\cup \Gamma_{n}\right) \cap(|z| \leqq R)$ is rectifiable and of length $\leqq k(R+1)^{c}$, where $k$ and $c$ are constants. Let $f_{n}(z)$ and $\phi_{n}(z)$ be holomorphic in $D_{n}, n \geqq 1$. Let $m(z)=$ dist $\left(z, \cup \Gamma_{n}\right)$. Let $A_{n}$ be a closed (but in general unbounded) subset of $D_{n}, n \geqq 1$. Let $a, \eta$, and $p$ be positive constants. Then there exists an entire function $F(z)$ such that for $z \in A_{n}, n \geqq 1,\left|f_{n}(z)-F(z)\right|<\eta\left\{1+m(z)^{-1}\right.$ $\left.\cdot(|z|+1)^{-p}\right\} \exp \left\{-a R_{\phi_{n}}(z)\right\}$. An appropriate choice of $\phi_{n}$ shows that this estimate is essentially the "best possible." (Received July 11, 1955.)

\section{8t. Szolem Mandelbrojt: Composition theorem on Fourier trans-} forms.

Let $F_{1}(z)=\int_{0}^{\infty} f_{1}(u) \exp (i u z) d u, F_{2}(z)=\int_{0}^{\infty} f_{2}(u) \exp (i u z) d u$, with $f_{1}, f_{2} \in L, f_{1}(u)$ $\cdot f_{2}(u) \equiv 0$. A relationship can be established between the singularities of $F_{1}(z)$ and those of $F_{2}(z)$ in the half-plane $y \leqq 0$. For instance, if some conditions are added concerning the growth of $F_{1}(z)$ in the half-plane $y \geqq d(d<0)$, when $|x| \rightarrow \infty$, then if $F_{1}(z)$ has, among its singularities, a pole on the real axis, $F_{2}(z)$ cannot have one, and only one, singularity in this same half-plane $(y \geqq d)$. (Received November 4,1955 .)

209. Szolem Mandelbrojt: Fourier transforms and functions holomorphic in a half-plane.

Consider the two equations (i) $\int K(y-x) F(x) d x=0$, (ii) $\int K(y-x) d F(x)=0$. In (i) suppose $K \in L, F \in B$, with $M=$ essent. max. of $|F|$. In (ii) suppose $K$ continuous, with $\sum_{n>-\infty} \operatorname{Max}_{x} \in[n, n+1]|K(x)|<\infty, F$ locally of bounded variation, with $\int_{n}^{n+1}|d F|<C$ (C independent of $\left.n\right)$. In (i) and (ii) suppose $k(u) \neq 0$ for $u<-h$, where $k$ is the Fourier transform of $K$ (in (ii): $h \geqq 0$ ). Then, in case (i), there exists a function $F_{0}(z)$, holomorphic for $y>0(z=x+i y)$, such that for each $N>0$ : (iii) $\lim _{y=0} \int_{-N}^{n} \mid F_{0}(x$ $+i y)-F(x) \mid d x=0$, with $\left|F_{0}(z)\right| \leqq M \cdot \exp (h y)(y>0)$. In case (ii) there exists a function $F_{0}(z)$, holomorphic for $y>0(z=x+i y)$, such that (iii) is satisfied, and for each 
$c>0:\left|[(\exp (i c z)-1) / z] F_{0}(z)\right| \leqq \operatorname{Sup} \xi|[(\exp (i c \xi)-1) / \xi] F(\xi)| \exp (h y)(y>0) .(\operatorname{Re}-$ ceived November 4, 1955.)

\section{0t. E. P. Merkes: A note on harmonic functions.}

Contained in this paper is a generalization of a theorem of Beckenbach on the definition of a harmonic function (Bull. Amer. Math. Soc. vol. 51 (1945) pp. 240-245). The proof of this result is made a very simple consequence of the well-known generalizations of the Looman-Menchoff Theorem by means of the concepts and notations introduced by $\mathrm{E}$. Kasner in connection with polygenic function theory. Extensions are made to $q$-harmonic functions. (Received November 14, 1955.)

211. E. P. Miles, Jr. (p) and Ernest Williams: The Cauchy problem for linear partial differential equations with restricted boundary conditions. III.

The authors apply their theorem of I (Abstract 62-1-103) to the damped wave equation (A): $\left[\nabla^{2}-\left(D_{t}^{2}-k^{2}\right)\right] u=0$ subject to the initial conditions $u(x, y, z, 0)=F(x, y, z)$, $u_{t}(x, y, z, 0)=0$ where $\nabla^{2 j} F \neq 0, \nabla^{2 j+2} F=0$. The solution is seen to be of the form (B), $u=\sum_{s=0}^{i} \nabla^{2 s} F \cdot u_{s}$ where the $\left\{u_{s}\right\}$ are given as follows: $u_{0}=\cosh k t, u_{1}=(t \sinh k t) / 2 k$ and $u_{s}=(-1)^{s} /(2 s) ! ! k^{2 s}\left\{\sum_{n=1}^{[s / 2]}\left[(2 s-2 n-1) !(k t)^{2 n}\right.\right.$ cosh $\left.k t / 2^{s-2 n}(s-2 n) !(2 n-1) !\right]$ $\left.-\sum_{n=1}^{[(s+1) / 2]}\left[(2 s-2 n) !(k t)^{2 n-1} \sinh k t / 2^{s-2 n+1}(s-2 n+1) !(2 n-2) !\right]\right\}$. For (A) with initial conditions $u(x, y, z, 0)=0, u_{t}(x, y, z, 0)=G(x, y, z)$, and $G$ polyharmonic of order $r+1$, the same methods lead to a solution of type $V=\sum_{s=0}^{r} \nabla^{28} G \cdot v_{s}$ which can be added to (B) to provide the solution of (A) for polyharmonic initial values for $u$ and $u_{t}$. The $\left\{u_{s}\right\}$ given above with the factor $(-1)^{s}$ deleted enable us to obtain analogous solutions of the Cauchy problem for the special Helmholtz Equation $\left(\nabla^{2}-k^{2}\right) u=0$. (Received November 14, 1955.)

212. L. E. Payne and H. F. Weinberger (p): Lower bounds for eigenvalues of elastically supported membranes.

The eigenvalues $\lambda_{1}(k) \leqq \lambda_{2}(k) \leqq \cdots$ of the problem $\Delta u+\lambda u=0$ on a bounded two-dimensional domain $R$ with $\partial u / \partial n+k u=0$ on the boundary of $R$ are considered. Let $\mu_{1}(k, L) \leqq \mu_{2}(k, L) \leqq \cdots$ be the eigenvalues of the one-dimensional problem $v^{\prime \prime}+\mu v=0, v^{\prime}(0)-k v(0)=0, v^{\prime}(L)+k v(L)=0$. (They are the solutions of a simple transcendental equation.) Let $L$ be the maximum length of any line segment contained in $R$ and parallel to the $x$-axis (the $x$-direction is, of course arbitrary.) Then it is shown that $\lambda_{1}(k) \geqq \mu_{1}(k, L)$. While equality is not attained except in the trivial case $k=0$, it is approximated with arbitrary accuracy for a sufficiently narrow rectangle with its long side in the $x$-direction. Furthermore, if $R$ is symmetric with respect to both the $x$ and $y$ axes, if the intersection of $R$ with any line in the $x$ or $y$ direction is simply connected, and if $L$ is maximum length of such intersections, then $\lambda_{2}(k)$ $\geqq \inf \left\{\mu_{2}(k, L), \lambda_{1}(\infty)\right\}$. For $k=0$, this gives a lower bound for the principal frequency of a free membrane. Equality is attained in this case for an arbitrary rectangle. (Received October 24, 1955.)

213. G. O. Peters: Euler polynomials and numbers of the second kind.

Nörlund, in his Verlesungen über Differenzenrechnung, defines Euler polynomials and numbers of higher order and of negative order. Interchanging the roles of $\Delta$ and $D$ and replacing powers of $x$ by factorials of $x$, the author calls a new set of poly- 
nomials, Euler polynomials of the second kind. The author first defines a new operator $\neg$ (Daleth) where $\urcorner=1+D / 2$ then denotes Euler polynomials and numbers of the second kind, of order $n$ and degree $\nu$, by $e_{\nu}^{n}(x), e_{\nu}^{n}$, and $c_{\nu}^{n}$ respectively, defined by: $e_{\nu}^{0}(x)=x^{(\nu)}=x(x-1)(x-2) \cdots(x-\nu+1) ; \quad 7 e_{\nu}^{\prime}(x)=x^{(\nu)} ; \quad 7^{n} e_{\nu}^{n}(x)=x^{(\nu)} ; \quad e_{\nu}^{n}(x)$ $=\sum_{i=0}^{\nu} \nu c_{i}\left(e_{i}^{n} / 2^{i}\right)(x-n / 2)^{(\nu-i)}=\left(e^{n} / 2+x-n / 2\right)^{(\nu)} ; \quad e_{\nu}^{n}(x)=\sum_{i=0}^{\nu} \nu c_{i}\left(c_{i}^{n} / 2^{i}\right) x^{(\nu-i)}$ $=\left(c^{n} / 2+x\right)^{(\nu)} ; \nu=0,1,2, \cdots ; n=0, \pm 1, \pm 2, \cdots$. These are Appel polynomials of the second kind since $\Delta e_{\nu}^{n}(x)=\nu e_{\nu_{1}}^{n}(x)$. Properties of these polynomials are found. Formulas, involving Sterling's numbers of the second kind, are found for evaluating the Euler numbers of the second kind of higher order. The symmetry of the polynomials is found to be given by $e_{\nu}^{n}(x)=(-1)^{\nu} e_{\nu}^{n}(\nu+n-1-x)$. (Received November 14, 1955.)

\section{Alexander Peyerimhoff: Summability factors for Cesdro's method.}

It is well known that a sequence $\left\{\epsilon_{n}\right\}$ transforms every $C_{\alpha}$-summable series $\sum a_{n}(\alpha \geqq 0)$ into a $C_{\beta}$-summable series $\sum a_{n} \epsilon_{n}(\beta \geqq 0)$ if and only if $\epsilon_{n}=O\left(n^{\beta-\alpha}\right)$, $\epsilon_{n}=O(1)$ and $\sum(n+1)^{\alpha}\left|\Delta^{\alpha+1} \epsilon_{n}\right|<\infty$ (Theorem of I. Schur). In this report a new characterization of these summability factors $\epsilon_{n}$ is given: A sequence $\left\{\epsilon_{n}\right\}$ has the above property if and only if (i) $\epsilon_{n}=O(1)$ and (ii) $\sum(n+1)^{\alpha}\left|\Delta^{\alpha+1} \epsilon_{n} \delta_{n}\right|<\infty$ for all sequences $\delta_{n}=o(1)$ and $\sum(n+1)^{\beta}\left|\Delta^{\beta+1} \delta_{n}\right|<\infty$. By use of this characterization it is possible to simplify the hitherto existing proofs of Schur's theorem. There are corresponding results for summability factors of the types $\left(\left|C_{\alpha}\right|,\left|C_{\beta}\right|\right)$ and $\left(C_{\alpha},\left|C_{\beta}\right|\right)$. (Received November 14, 1955.)

215. T. J. Pignani (p) and W. M. Whyburn: Differential systems with interface and general boundary conditions.

The general linear differential system of the $n$th order is studied in cases where the solutions are required to satisfy certain interface conditions as well as linear boundary conditions of quite general type. Existence of nonsingular solutions for the homogeneous system is established for cases where singular coefficient matrices occur in the interface conditions. Through use of these solutions of the homogeneous system, and functions which generalize the Green's functions of classical analysis, solutions are obtained for a large class of nonhomogeneous systems. Some of the results are extended to cases where the set of interface points is infinite. (Received September $6,1955$.

\section{6t. Pasquale Porcelli: A Stieltjes type of inversion.}

The following theorems are proved: Theorem $A$. Let $g$ be a nondecreasing function on $[0,1]$ and $F(z)=\int_{0}^{1}(1 / 1+z t) d g(t)$ for $z \notin[-\infty,-1]$, then $g(u)-g(v)=(-1 / \pi)$ $\cdot \lim _{y \rightarrow 0_{+}} \int_{v}^{u} 1 / x I_{m} F(-1 / x+i y) d x$, where $0<v<u<1, g$ is continuous at each of $u$ and $v$, and $I_{m} F(z)$ is the imaginary part of $F(z)$. Theorem B. If $F$ and $g$ satisfy the integral equation of Theorem $\mathrm{A}$, then there exists a nonnegative function $f$ on $(0,1)$ such that $f$ is Lebesgue summable on each subinterval $[v, u]$ of $(0,1)$ and $(-1 / \pi) \lim _{y \rightarrow 0}+1 / \pi$ - $I_{m} F(-1 / x+i y)=f(x)$ almost everywhere; moreover, $g^{\prime}(x)=f(x)$ almost everywhere. (Received November 14, 1955.)

217t. John Raleigh: The Fourier coefficients of the invariants $j\left(2^{1 / 2} ; \tau\right)$ and $j\left(3^{1 / 2} ; \tau\right)$.

Two infinite Fuchsian groups of linear transformations, $G\left(2^{1 / 2}\right)$ and $G\left(3^{1 / 2}\right)$, are 
considered: $G\left(2^{1 / 2}\right)$ with generators $S(\tau)=\tau+2^{1 / 2}, T(\tau)=-1 / \tau ; G\left(3^{1 / 2}\right)$ with generators $S(\tau)=\tau+3^{1 / 2}, T(\tau)=-1 / \tau$. Both groups possess invariants; respectively: $j\left(2^{1 / 2} ; \tau\right)$ $=x^{-1}+\sum_{n=0}^{\infty} c_{n} x^{n}, x=\exp \left(2 \pi i \tau / 2^{1 / 2}\right), j\left(3^{1 / 2} ; \tau\right)=x^{-1}+\sum_{n=0}^{\infty} a_{n} x^{n}, x=\exp \left(2 \pi i \tau / 3^{1 / 2}\right)$. It is required to determine the coefficients of the above expansions. The Hardy-Littlewood method is employed for the purpose, and the procedure is an extension of the one used by $\mathrm{H}$. Rademacher for the Fourier coefficients of the modular invariant $j(1 ; \tau)$. Convergent series for the $c_{n}$ 's and the $a_{n}$ 's are obtained: $c_{n}=\left(2 \pi / n^{1 / 2}\right) \sum_{\nu=1}^{\infty} A_{2 \nu}(n) / 2_{\nu}$ $\cdot I_{1}\left(2 \pi n^{1 / 2} / \nu\right)+\left(2 \pi /\left(2 n^{1 / 2}\right)\right) \cdot \sum_{\nu=1}^{\infty} A_{2 \nu-1}((1-\nu) n) / 2 \nu-1 \cdot I_{1}\left(2 \pi(2 n)^{1 / 2} / 2 \nu-1\right)$, $a_{n}=\left(2 \pi / n^{1 / 2}\right) \sum_{\nu=1}^{\infty} A_{3 \nu}(n) / 3 \nu \cdot I_{1}\left(4 \pi n^{1 / 2} / 3 \nu\right)+\left(2 \pi /(3 n)^{1 / 2}\right) \sum_{\nu=1}^{\infty} A_{3 \nu-1}(\nu n) / 3 \nu-1$ $\cdot I_{1}\left(4 \pi(3 n)^{1 / 2} / 3(3 \nu-1)\right)+\left(2 \pi /(3 n)^{1 / 2}\right) \sum_{\nu=1}^{\infty} A_{3 \nu-2}((1-\nu) n) / 3 \nu-2 \cdot I_{1}\left(4 \pi(3 n)^{1 / 2} / 3(3 \nu-2)\right)$, where $n \geqq 1, A_{k}(m)=\sum^{\prime} \exp \left(-2 \pi i\left(m h+h^{\prime}\right) / k\right), h h^{\prime} \equiv-1(\bmod k), \sum^{\prime}$ indicates the sum with respect to $h$ from 0 to $k-1$ with $(h, k)=1$ and $I_{1}(z)$ is the Bessel function of first order with purely imaginary argument. Moreover, algebraic equations are established relating $j(1 ; \tau)$ to $j\left(2^{1 / 2} ; \tau\right)$ or to $j\left(3^{1 / 2} ; \tau\right)$ respectively. Such equations may be used to calculate the $c_{n}$ 's and the $a_{n}$ 's in closed form, for the first few $n$ 's and hence to verify, in part, the results obtained from the series. (Received November 14, 1955.)

\section{8t. M. O. Reade: Radial limits of certain subharmonic functions.}

We prove the following theorem, which represents a generalization of a result due to Beckenbach and Rado (Trans. Amer. Math. Soc. vol. 35 (1933) pp. 648-661). If $p(x, y)$ is bounded and of class $P L$ in the disc $|z|=|x+i y|<1$, and if $\lim _{r \rightarrow 1} p(z)$ $=\lim _{r \rightarrow 1} p\left(r e^{i \theta}\right)=0$ for a set of $\theta^{\prime} s$ of positive (linear) measure, then $p(z) \equiv 0$. The technique used is that due to Caratheodory (Funktionentheorie, Basel, 1950, vol. II, p. 46). (Received November 15, 1955.)

\section{W. T. Reid: Oscillation criteria for linear differential systems with complex coefficients.}

For a self-adjoint second order linear vector differential equation $L[u] \equiv\left(R(x) u^{\prime}\right.$ $+Q(x) u)^{\prime}-\left(Q^{*}(x) u^{\prime}+P(x) u\right)=0$ with complex-valued coefficients there are presented various general variational criteria for oscillation and nonoscillation, corresponding to known results for the case of such systems with real coefficients. Certain specific criteria for a scalar second order differential equation are generalized to the above vector equation. The results established for the self-adjoint equation $L[u]=0$ are used to deduce sufficient conditions for a general vector differential equation of the form $A_{2}(x) u^{\prime \prime}+A_{1}(x) u^{\prime}+A_{0}(x) u=0$ to be nonoscillatory. (Received November 14, 1955.)

\section{0t. H. J. Reiter: $L^{1}$-spaces on groups. III.}

Let $g$ be a discrete abelian group, $B$ a Banach space. Define the space $L_{B}^{1}(g)$ as the space of all functions on $g$ with values in $B: f=\left(f_{k}\right), k \in g, f_{k} \in B$, with $L^{1}$-norm $\left\|_{f}\right\|_{1}$ $=\sum_{k \in o}\left\|f_{k}\right\|<\infty$, where $\|\cdot\|$ denotes the norm in B. Theorem: inf $\left\|f_{k}-\sum_{r=1}^{R} b_{r} f_{k+n_{r}}\right\|_{1}$ $=\left\|\sum_{k \in o} f_{k}\right\|$, where $R$ ranges over all positive integers, the coefficients $b_{r}$ range over all complex numbers satisfying $\sum_{r=1}^{R} b_{r}=0$, and the elements $n_{r}$ over the group $g$. Application: $B$ is the space of all complex-valued continuous functions $f(t)$ on $[0,1]$, with norm $\|f\|=\max _{0} \leqq_{t} \leqq_{1}|f(t)|, g$ theadditive group of integers. Then the space $W$ of all continuous functions $f(x),-\infty<x<\infty$, with norm $\sum_{n-\infty}^{\infty} \max _{n} \leqq_{x} \geqq_{n+1}|f(x)|$ $<\infty$, considered by N. Wiener (cf. N. Wiener, Tauberian theorems, Ann. of Math. vol. 33 (1932) pp. 1-100) is a (closed) subspace of $L_{B}^{1}(g)$. Let $W_{0}$ consist of all functions in $W$ such that $\sum_{n=-\infty}^{\infty} f(x+n)=0$. Then the quotient-space $W / W_{0}$ is isomorphic and 
isometric to the space of all continuous functions of period 1 under the mapping $f(x) \rightarrow T f(x)$, where $T f(x)=\sum_{n=-\infty}^{\infty} f(x+n)$; a corresponding result holds for subspaces of $W$ which are invariant under the translations $f(x) \rightarrow f(x+k), k$ an integer. The definition of the space $W$, and the result, may be extended to locally compact groups $G$ containing a discrete abelian subgroup $g$ such that the quotient-space $G / g$ is compact. (Received November 14, 1955.)

\section{1t. H. M. Schaerf: The equivalence of two continuity properties of} measurable functions. Preliminary report.

A measure space $(R, S, m)$ whose carrier is a general topological space is said to have the property of (a) Lusin or (b) Vitali-Caratheodory if the following statements hold, respectively: If $f$ is any measurable function on $R$, then (a) for every positive number $\epsilon$ and every measurable set $E$ there is a closed measurable set $F C E$ with $m(E-F)<\epsilon$ such that $f$ is continuous on $F$; (b) there are two monotone sequences of semi-continuous functions $\left\{l_{n}\right\}$ and $\left\{u_{n}\right\}$ which converge to $f$ almost everywhere and have the properties stated in the usual formulations of the Vitali-Caratheodory theorem in Euclidean spaces (e.g. in S. Saks, Theory of the integral, 2d edition, Theorem 7.6). Theorem: For every totally $\sigma$-finite measure space whose carrier is a general topological space the above two properties are equivalent. Criteria for the validity of generalizations of the Vitali-Caratheodory theorem to spaces without topological structure are also established. (Received November 14, 1955.)

222t. V. L. Shapiro: The symmetric derivative on the $(k-1)$-dimensional hypersphere.

Let $x$ be a point on the unit (k-1)-dimensional hypersphere $\Omega$ in Euclidean $k$ space, $k \geqq 3$, and let $\mu$ be a completely additive set function of bounded variation defined on the Borel sets of $\Omega$. Let $D(x, h)$ represent the spherical cap on $\Omega$ obtained by intersecting $\Omega$ with a sphere whose center is $x$ and radius is $2 \sin h / 2$, and let $|D(x, h)|$ be the $(k-1)$-dimensional volume of $D(x, h)$. Then $\mu$ will be said to have a symmetric derivative at $x$, designated by $\mu_{s}(x)$, if $|D(x, h)|^{-1} \mu[D(x, h)]$ tends to $\mu_{s}(x)$ as $h$ tends to zero. Let $S\left[d_{\mu}\right]=\sum_{n=0}^{\infty} Y_{n}(x)$ be the Stieltjes series of surface harmonics defined by $\mu$. Then it is shown in this paper that if $\mu_{s}\left(x_{0}\right)$ exists and is finite and $\mu$ satisfies the global condition $|\mu|\left[D\left(x_{0}^{\prime}, \epsilon\right)\right]=0$ for some $\epsilon>0$, where $x_{0}^{\prime}$ is the point diametrically opposite to $x_{0}$ and $|\mu|$ is the total variation of $\mu$, then $S\left[d_{\mu}\right]$ is summable $(C, \delta), \delta>(k-2 / 2)+1$, to $\mu_{s}\left(x_{0}\right)$. This result generalizes the well-known result for Fourier-Stieltjes series where $\delta>1$. In case the global condition is not satisfied, it is shown that $S[d \mu]$ is summable $(C, \eta)$ to $\mu_{s}\left(x_{0}\right)$ where $\eta>k-2$ for $k \geqq 4$ and $\eta>3 / 2$ for $k=3$. In the special case when $\mu$ is absolutely continuous and $Y_{n}\left(x_{0}\right)=O\left(n^{-1}\right)$, a necessary and sufficient condition that $S\left[d_{\mu}\right]$ converges at $x_{0}$ to the finite value $b$ is obtained also in this paper, namely that $\mu_{s}\left(x_{0}\right)$ exists and equals $b$. This fact generalizes a result previously obtained for Fourier series by Hardy and Littlewood. (Received November 9, 1955.)

\section{R. C. Thorne: Asymptotic expansions of Legendre functions.}

Although asymptotic expansions for the Legendre functions $P_{n}^{-m}(x), Q_{n}^{-m}(x)$ have been given for $m$ fixed and $n \rightarrow \infty$ by Olver (International Mathematical Congress, Amsterdam, 1954, vol. II), no expansions have been derived for both $n$ and $m$ large and positive. For this case, three sets of expansions in ascending powers of $u^{-1}(u$ $=n+1 / 2)$ are developed here. The ratio $0<\alpha=m / u<1$ is fixed and $u \rightarrow \infty$. The first expansions, involving exponential functions, are not valid over the transition point 
$x=\beta=\left(1-\alpha^{2}\right)^{1 / 2}$. The second set of expansions, obtained by application of Olver's theory (Phil. Trans. Roy. Soc. London, Ser. A. vol. 247, pp. 307-327), are in terms of Airy functions and are valid over the domain $D$ consisting of the whole $x$ plane (cut from +1 to $-\infty$ ) except for a pear shaped domain surrounding $x=-1$ and the strip $(S$, say) $-\delta \leqq \operatorname{Im}(x)<0,|\operatorname{Re}(x)|<\beta ; \delta$ small $>0$. Expansions valid in $D+S$ are obtained by using Bessel functions $\operatorname{Im}(u t), \mathrm{Km}(u t), t \equiv t(x)$. This theory is new and differs from that of Cherry (Trans. Amer. Math. Soc. vol. 68 (1950) pp. 253-257). Also, $P_{n}^{-m}(x)$ is exponentially small as $n \rightarrow \infty$ in a pear shaped region surrounding $x=1$, oscillatory for $x$ real and $|x|<\beta$, and exponentially large elsewhere. Related properties exist for $Q_{n}^{-m}(x)$ as $n \rightarrow \infty$. (Received December 1, 1955.)

\section{H. S. Wall: Partially bounded continued fractions.}

For each complex number sequence $a, f(a)$ denotes the continued fraction $1 / 1$ $+a_{1} / 1+a_{2} / 1+\cdots$. The statement that $f(a)$ is partially bounded means that the sequence $a$ has a bounded infinite subsequence. Theorem. If there is a nonnegative integer $k$ such that the subsequence $\left\{f_{i}\right\}_{i-k}^{\infty}$ of the sequence of approximants of the partially bounded continued fraction $f(a)$ is bounded and the even (odd) part of $f(a)$ converges and has the value $v$, then there exists an infinite subsequence of the sequence of approximants of the odd (even) part of $f(a)$ which converges to $v$. Corollary. If the even and odd parts of the partially bounded continued fraction $f(a)$ converge, then $f(a)$ converges. (Received October 31, 1955.)

\section{ApPlied Mathematics}

\section{5t. R. W. Bass: Improved on-off missile stabilization.}

A new synthesis procedure for on-off control systems is presented, by means of which the delays due to time-lag and hysteresis in the relay or contactor can be completely eliminated. This is readily effected by adding feedback from the on-off element itself to the conventional error and error-derivative feedbacks. By generalization (to inhomogeneous piecewise-linear difference-differential equations) of the functional relationship $E(t+s)=E(t) E(s)$ (which is satisfied by any fundamental matrix of solutions of a system of homogeneous linear differential equations with constant coefficients), an explicit algorithm is developed, according to which the ideal feedback coefficients of a "perfectly" stabilized servo-system can be read off directly from the equations of the corresponding conventional system. The use of this algorithm is illustrated by application to three second order systems, and one third order system, of one degree of freedom, and to one second order system with three degrees of freedom. The results are applied to several specific relay servomechanisms and missile control systems. (Received November 14, 1955.)

\section{6t. Stefan Bergman: On a class of subsonic flows.}

The author considers subsonic flow patterns in a channel $C$ bounded by $[y=0$, $-\infty<x<\infty]$ on one side, the segments $\left(-\infty A_{0}\right)=[y=-c=$ const., $c>0,-\infty<x$ $<0],\left(A_{n-1} A_{n}\right)=\left[x=y \cos \tau_{n}+a_{n}\right], n=1,2, \cdots, N, \tau_{n}=$ const., $0<\tau_{1} \cdots<\tau_{n-1}$ $<\tau_{n}<\cdots \tau_{N}<\pi / 2$, and the segment $\left(A_{N} \infty\right)$ of a free boundary on the other side. The image of the flow in the pseudo logarithmic plane (see Bergman, Proceedings of Symposium in Applied Mathematics, vol. $6, \S 4 \mathrm{ff}$.) is a half strip bounded by the segment $\left(a_{N-1} a_{N}\right)=\left[\theta=\tau_{N}, \lambda<\lambda_{1}\right],\left[\lambda=\lambda_{1}, 0 \leqq \theta<\tau_{N}\right]$ (which is the image of the free boundary), $[\theta=0, \lambda=\lambda(0)],\left[\theta=0, \lambda(0) \leqq \lambda \leqq \lambda_{1}\right]$, and $(N-1)$ slits $S_{n}=\left[-\infty<\lambda<\alpha_{n-1}\right.$, $\left.\theta=\tau_{n}\right], n=1,2, \cdots, N-1 . \lambda^{(0)}$ is the image of $[y=-\infty]$ and the slits are images of 
sides $\left(A_{n-1} A_{n}\right), n=1,2, \cdots, N-1$. The stream function $\psi(\lambda, \theta)$ satisfies a linear differential equation $L(\psi)=\psi_{\lambda \lambda}+\psi_{\theta \theta}+N(\lambda) \psi=0$ (see Bergman, Journal for Rational Mechanics and Analysis vol. 4, \$2). Using the theory of linear integral equations the author shows that for every set of slits $S_{n}$ and every $\lambda^{(0)}$ (satisfying certain conditions) there exists the desired stream function $\psi(\lambda, \theta), \mathrm{L}(\psi)=0$. Using the theory of orthogonal functions (see Bergman and Schiffer, Duke Math. J. vol. 15, in particular \$7, p. $560 \mathrm{ff}$.) one obtains a representation for the stream function $\psi(\lambda, \theta)$. (Received December 13, 1955.)

\section{L. A. Colquitt: $A$ modified iteration procedure.}

Suppose that the equation $x=f(x)$ has a unique root, and suppose that $f^{\prime}(x)$ satisfies the inequality $-a<f^{\prime}(x)<-1$ for all $x$, so that the conventional iteration fails to converge. The following modified iteration procedure converges for this case. Let $x_{0}$ be arbitrary, and define $x_{2 n-1}=f\left(x_{2 n-2}\right) ; x_{2 n}=1 / 2 n \sum_{i=0}^{2 n-1} x_{i}, n=1,2, \cdots$. In other words, substitutions alternate with averagings. If $x$ is the desired root of the given equation, it can be shown that $x-x_{2 n}=1 / 2 n\left(x-x_{0}\right)\left[1+f^{\prime}\left(x_{0}^{\prime}\right)\right] \prod_{i=1}^{n-1}\{1+1 / 2 i[1$ $\left.\left.+f^{\prime}\left(x_{2 i}^{\prime}\right)\right]\right\}$, where $x_{2 i}^{\prime}$ lies between $x$ and $x_{2 i}$. From this relation it follows that $\lim _{n \rightarrow \infty}\left(x-x_{2 n}\right)=0$. Even if $f(x)$ is such that $-1<f^{\prime}(x)<0$, so that the conventional iteration converges, it may do so only slowly if $f^{\prime}(x)$ is close to -1 . A procedure similar to that used above will accelerate the convergence in this case. (Received November 14, 1955.)

\section{A. H. Diamond: Certain classes of finite sequential machines} with distinguished states.

In a paper entitled Gedanken-experiments on sequential machines, which will appear in the Automata Studies, E. F. Moore develops the theory of strongly connected machines, that is, machines having the property that for any pair of states of the machine there exist input sequences which will take the machine from either of the two states to the other. In this paper some of Moore's results are extended to certain classes of quasi strongly connected machines with distinguished states. (Received November 14, 1955.)

229t. H. H. Goldstine and Joseph Gillis: The stability of superposed fuids.

Two homogeneous, incompressible, nonviscous fluids, which may have different densities, are superposed in equilibrium one above the other between a pair of infinite, parallel, vertical walls. The walls are defined by $x=0, x=\pi$, while the equilibrium position of the interface between the fluids, supposed horizontal, is given by $y=0$. Equilibrium is perturbed and the subsequent motion studied, with particular reference to the question of stability. The configuration of the interface at time $t$ is expressed as a double series $y=\sum_{m, n} \eta_{m, n}(t) \alpha^{m} \cos n k x$ where $\alpha$ is a perturbation parameter and $k$ the mode of the perturbation. The coefficients $\eta_{m, n}(t)$ satisfy linear differential equations of the second order which are obtainable by means of an algorithm. Some general results are derived relating to the functions $\eta_{m, n}(t)$ and they are calculated as explicit functions of $t$ for $1 \leqq m \leqq 5$ and all relevant $n$. The resulting approximation to $y$ is compared with the known experimental facts in some special cases. (Received November 14, 1955.)

230. T. P. Higgins (p) and W. T. Guy, Jr.: On the Hankel transform. II. 
The Hankel integral transform of the $n$th derivative of a function is used to establish some new relations involving Meijer's $G$-functions. (Received November 14, 1955.)

\section{Ernest Ikenberry: The collision integral for Maxwellian mole-} cules; elementary spherical moments.

In this paper there are obtained the collision integrals $C\left(Y_{\bullet}\right)$ for homogeneous spherical harmonics $Y_{s}(c)$, for Maxwellian molecules. In the notation adopted by Ikenberry and Truesdell (Journal of Rational Mechanics and Analysis, forthcoming), $C(Q)=\int_{\infty} d c \int_{\infty} d c^{*} \int_{0}^{\infty} 2 v b d b \int_{0}^{2 \pi} d \in\left\{Q^{\wedge}-Q\right\} F F^{*}$. Let elementary spherical moments $S_{s}$ of order $s$ be defined by $S_{s}=m \int_{\infty} C^{s} F d c$, where $C^{s} \equiv\left(c_{3}+i c_{1} \cos \alpha+i c_{2} \sin \alpha\right)^{s}$ is a homogeneous spherical harmonic of degree $s$. Then $m \rho C\left(C^{s}\right)=-\sum_{i_{-0}}^{i} C_{s(j)} S_{j} S_{s-j}$ where $2^{s-1} C_{s(j)}=n \sum_{l=0}^{i} \sum_{k=j-l}^{s-l}(-1)^{i+l}\left(C_{k}^{s}\right)\left(_{l}^{(-k}\right)\left(_{j-l}^{k}\right) B_{k}$, with $B_{k}=2 \pi \int_{0}^{\infty}\left\{1-P_{j}(\cos \phi)\right\} v b d b$. From this, the value of $C\left(Y_{s}\right)$ for any homogeneous spherical harmonic $Y_{s}(\mathrm{c})$ of degree $s$ is obtainable: $C\left(Y_{s}\right)=\int_{0}^{2 \pi} C\left(C^{s}\right) f_{8}(\alpha) d \alpha$, where $f_{8}(\alpha)$ is a uniquely determined trigonometric polynomial of degree $s$ such that $Y_{s}(\mathrm{c})=\int_{0}^{2 \pi} C^{8} f_{s}(\alpha) d \alpha$. (Received November 10, 1955.)

\section{J. R. Isbell (p) and W. H. Marlow: Programming games.}

A linear fractional function in $n$-space can be factored into a linear transformation upon the plane and a plane linear fractional function. Thus a simple computational method for linear fractional programming is found. Further, certain tactical games involving strategic choice of distributions of fire of weapons over several targets, assuming attrition is governed by certain generalized Lanchester equations (due to R. N. Snow, Rand publication RA-15078), have payoff functions linear fractional in both players' strategies together (not merely bilinear fractional). Thus a simple computational method for solving these games is found. Both authors supported in part by the Office of Naval Research; the first is a private in the Army Ordnance Corps. (Received November 9, 1955.)

\section{H. A. Osborn: A new technique for eigenvalue problems.}

If $\phi(t)$ is a sufficiently smooth positive function of $t$ in $[0,1]$ the following method furnishes an efficient technique for computing the lowest eigenvalue $\lambda$ of $u^{\prime \prime}+\lambda \phi(t) u$ $=0, u(0)=u(1)=0$. For any $s \in[0,1]$ and any $v \geqq 0$ set $f(s, v)=\min \int_{0}^{s} w^{\prime 2}(t) d t$ subject to $w(0)=0, w(s)=v$, and the normalization $\int_{0}^{a} \phi(t) w^{2}(t) d t=s^{3}$, assuming that the minimum is attained for some twice-differentiable function $w(t)$. Then for any $\sigma \in(0,1)$ we have the functional inequality $f(s, v) \geqq \min \left\{\int_{\sigma}^{*} w^{\prime 2}(t) d t+r^{-2} f\left(\sigma, r v^{*}\right)\right\}$, where the minimum is taken over $v^{*}>0$ and $w(t)$ for $t \in[\sigma, s]$ subject to $w(\sigma)=v^{*}, w(s)=v$, and where $r$ is a factor depending on $w(t)$ which accounts for the renormalization to the interval $[0, \sigma]$. The two sides of the inequality actually differ only by $o(s-\sigma)$. Replacing $\int_{\sigma}^{s} w^{\prime 2}(t) d t$ and $r$ by approximations correct to $o(s-\sigma)$ one finds a difference equation which permits the computation of $\lambda=f(1,0)$ to within $O(h)$, where $h$ is the difference interval. The initial values of the difference scheme do not depend on a trial value of $\lambda$. (Received November 10, 1955.)

234. H. H. Rachford (p) and Jim Douglas, Jr.: On the numerical solution of heat condition problems in two and three space variables.

The objective of this paper is to introduce a new implicit finite difference method for obtaining approximate solutions of both transient and permanent heat conduction 
problems in two or three space variables. Let $f_{i j k n}=f(i \Delta x, j \Delta y, k \Delta z, n \Delta t)$ and $\Delta_{x}^{2} f_{i j k n}$ $\left.=\left(f_{i+1, j, k, n}-2 f_{i j k n}+f_{i-1, j, k, n}\right) / \Delta x\right)^{2}$. The difference analogue of the parabolic case with three space variables is the following three step procedure: $\Delta_{x}^{2} w_{i, j, k, n+1}^{*}+\Delta_{y}^{2} w_{i j k n}$ $+\Delta_{z}^{2} w_{i j k n}=\left(w_{i, j, k, n+1}^{*}-w_{i j k n}\right) / \Delta t, \quad \Delta_{y}^{2} w_{i, j, k, n+1}^{* *}=\Delta_{y}^{2} w_{i j k n}+\left(w_{i, j, k, n+1}^{* *}-w_{i, j, k, n+1}^{*}\right)^{\nu} / \Delta t$, $\Delta_{z}^{2} w_{i, j, k, n+1}=\Delta_{z}^{2} w_{i j k n}+\left(w_{i, j, k, n+1}-w_{i, j, k, n+1}^{*}\right) / \Delta t$. It is proved that this method is stable and that its solution for the initial- and boundary-value problem converges in the mean to that of the heat flow equation with the error being $O\left((\Delta x)^{2}+(\Delta t)^{2}\right.$ $\left.+(\Delta x)^{4}(\Delta t)^{-1}\right)$. The method is then applied to the elliptic case by considering the time step as an iteration parameter. A sequence of parameters is constructed so that the number of calculations necessary to reduce the norm of the difference between the iterated solution and the solution of $\Delta_{x}^{2} w_{i j k}+\Delta_{y}^{2} w_{i j k}+\Delta_{z}^{2} w_{i j k}=0$ by the factor $\exp (-Q)$ is $O(Q N \log N)$, where $N$ is the number of interior points in the lattice. The two space variable case is treated by the analogous two step procedure. (Received November 7, 1955.)

235t. H. E. Salzer: Osculatory extrapolation and a new method for the numerical integration of differential equations.

One may define "confluent binomial coefficients," or multipliers of the function and its derivative, which play a role in both extrapolation and checking similar to that of binomial coefficient multipliers for the function alone. The coefficients $C_{k}$ and $D_{k}$ in the $n$-point osculatory extrapolation formula of $(2 n-1)$ th degree accuracy. viz. (1) $f_{[n / 2]+1}=\sum_{k=-[(n-1) / 2]}^{k-[2]}\left(C_{k} f_{k}+h D_{k} f_{k}^{\prime}\right)+R_{2 n}$, are tabulated exactly for $n=2(1) 11$, with the factor $F_{n}$ where $R_{2 n} \cong F_{n} \cdot \Delta^{2 n} f$. Comparison with ordinary extrapolation and checking by differencing shows advantages in (1) due to the smallness of $F_{n}$ as $n$ increases. The most important use of (1) is in providing, for the lower values of $n$, a new and more accurate set of formulas for the stepwise numerical integration of $y^{\prime}=\phi(x, y)$. Because of the error in $f_{[n / 2]+1}$ due to the magnitude of $C_{k}$ and $D_{k}$, (1) is used in conjunction with an entirely new type of refining formula (2) $f_{[n / 2]+1}$ $=\sum_{k=-[(n-1) / 2]}^{k-[n / 2]} A_{k} f_{k}+h \sum_{k=-[(n-1) / 2]}^{k-[n / 2]+1} B_{k} f_{k}^{\prime}$, i.e., " $n$ 1/2-point" osculatory type based upon $f$ and $f^{\prime}$ at $n$ points and $f^{\prime}$ at the $(n+1)$ th. The coefficients $A_{k}$ and $B_{k}$ in (2) were found up to $n=5$ (10th degree accuracy). For complex integration of differential equations of the form $w^{\prime}=\phi(z, w)$, over a Cartesian grid, oscula tory extrapolation and refining formulas analogous to (1) and (2) were derived, of 7 th and 8 th degree accuracy respectively, based upon $f$ and $f^{\prime}$ at the corners of a square and the extra $f^{\prime}$ at each of the four closest points, which is sufficient to continue the integration until all the desired grid points are included. (Received October 7, 1955.)

236. G. L. Spencer, II: A method for solving mixed initial problems for quasi-linear hyperbolic systems.

The method presented solves a subclass of the mixed initial problems discussed by Courant and Lax (On nonlinear partial differential equations with two independent variables, Courant and Lax, "Communications on Pure and Applied Mathematics," vol. II, 1949). It also solves a class of singular problems not covered by Courant and Lax. (Received November 14, 1955.)

237t. G. L. Thompson: On the solution of a game-theoretic problem.

During the consideration of a generalization of von Neumann's model of an expanding economy the following, essentially game-theoretic, problem arose: Given real-valued matrices $A$ and $B$ with non-negative entries and such that $v(-A)<0$ and $v(B)>0$; set $M(\alpha)=B+\alpha(-A)$ and find an $\alpha$ so that $v(M(\alpha))=0$; then find a pair 
of probability vectors $(x, y)$ such that $x B y>0$ and $x$ is optimal for the maximizing player and $y$ is optimal for the minimizing player in the game $M(\alpha)$. It is shown that at least one solution to this problem exists. Elsewhere it has been shown that there are at most a finite number of $\alpha$ 's for which solutions exist. Our proof utilizes the minimax theorem from the theory of games in the strong form proved by $\mathrm{A}$. W. Tucker. Specifically, the concept of a central solution to a matrix game is used. Examples of solutions plus possible extensions of the theorem are also considered. (Received November 14, 1955.)

\section{Deonisie Trifan: Stress theory of plastic flow.}

General stress-strain relations for the stress theory of plastic flow of an incompressible, isotropic material exhibiting a gradual transition from the elastic to the plastic state are developed by using the stress-strain relation for simple tension as a model, resulting in $d s_{i j}=2 G_{0} d e_{i j}-G\left\{\partial g / \partial e_{i j}-\left(4 E_{2} E_{3} / 3\right) \partial g / \partial E_{3}^{2} \delta_{i j}\right\} d g$ for loading and $d s_{i j}=2 G_{0} d e_{i j}$ for unloading. The quantities $d s_{i j}$ and $d e_{i j}$ are differentials of stress and strain deviations respectively, while the functions $G$ and $g$ are positive definite functions of the strain invariants $E_{2}$ and $E_{3}^{2}$. The loading-unloading criterion is given by the differential of $g$. Conditions on $G$ and $g$ are obtained and a minimum principle for the differential of strain deviation introduced, namely: For a suitable set of differentials of strain $d e_{i j}^{\prime}$ (called admissible) and corresponding differentials of stress deviation $d s_{i j}^{\prime}$, the integral $(1 / 2) \int_{V} d e_{i j}^{\prime} d s_{i j}^{\prime} d V-\int_{S_{2}} d u_{i}^{\prime} d T_{i} d S$ is an absolute minimum for the actual differentials of stress and strain occurring in the body; the boundary condition $d T_{i}$ being given over $S_{2}$. Inverted stress-strain relations to the above are calculated, and a corresponding minimum principle for the differential of stress deviation given. (Received November 1, 1955.)

\section{GEOMETRY}

239. L. M. Blumenthal: Remarks on the fundamental theorem concerning metric arcs with zero curvature at each point.

The existing literature does not contain a proof of the theorem that a metric, ptolemaic arc with zero Menger curvature at each point is a metric segment, that is both complete-in-itself (in an obvious sense) and which is free from error and troublesome lacunae. The purpose of the present investigation is to supply such a proof. (Received November 14, 1955.)

240. S. S. Cairns: On the partition of the vertices of an $n$-cube by an $(n-1)$-plane.

The work is motivated by the problem of characterizing the convex of the vertices of the $n$-cube $0 \leqq x_{i} \leqq 1$ satisfying an inequality $\sum a_{i} x_{i}<c\left(a_{i}>0\right)$. A vertex $p$ is identified with the subset $J$ of $(1, \cdots, n)$ such that, at $p, x_{i}=1$ if $i \in J$, and $x_{i}=0$ otherwise. Given the $a$ 's, the plane $\pi(c): \sum a_{i} x_{i}=c$ induces a permutation $\Pi(a)$ of the set $\{J\}$ of all $2^{n}$ subsets of $(1, \cdots, n)$, defined by the order in which $\pi(c)$ passes through the vertices, regarded as elements of $\{J\}$, when $c$ increases from a negative value through the value $\sum a_{i}$. By minor adjustments of the $a$ 's it can be arranged that $\pi(c)$ never passes simultaneously through two vertices. Various general properties are established for the permutations thus generated, and a recurrent method with respect to $n$ is developed. Some questions of consistency of linear inequalities make the general step of the recurrency difficult. The step from $n=3$ to $n=4$ reveals that there are 336 possible permutations $\Pi(a)$ for the vertices of a 4-cube, 148 possible sets of vertices 
defined by $\sum a_{i} x_{i}<c$ and only 27 types of convex hulls of such sets, two convexes being of the same type if one can be carried into the other by a permutation of axes. (Received November 14, 1955.)

\section{N. A. Court: On four real mutually orthogonal spheres.}

Considering the sphere of similitude, the radical sphere, and the two spheres of antisimilitude of the given spheres taken two at a time, twenty four spheres are obtained which may be grouped into six tetrads such that the spheres in each tetrad are mutually orthogonal. If the given spheres are taken three at a time, four pairs of real points of intersection are obtained. Taking one and only one point in each pair, sixteen tetrahedrons are formed. All these tetrahedrons are isodynamic, and may be grouped into eight pairs of homological tetrahedrons, their planes of homology coinciding with the eight planes of similitude of the four given spheres, etc. It follows from the latter proposition that the problem: "Through four given points, taken three at a time, to pass four mutually orthogonal spheres" admits of no solution, unless the four given points form an isodynamic tetrahedron. If this condition is satisfied, the problem may, in general, have two solutions. (Received November 14, 1955.)

\section{M. K. Fort, Jr.: A geometric problem of S. Stein.}

S. Stein has proposed the following problem (Bull. Amer. Math. Soc. Research Problem 61-5-25): Let $J C R_{2}$ be a rectifiable Jordan curve, with the property that for each rotation $R$, there is a translation $T$, depending on $R$, such that $(T R J) \cap J$ has a nonzero length. Must $J$ contain the arc of a circle? If "length" is interpreted to mean the one-dimensional Lebesque measure which is induced on $J$ by arclength, then an example shows that $J$ need not contain the arc of a circle. The following theorem is then proved: If $J C R_{2}$ is a (not necessarily rectifiable) Jordan curve, with the property that for each of an uncountable number of rotations $R$ about some point there is a translation $T$, depending on $R$, such that $(T R J) \bigcap J$ has a nondegenerate component, then $J$ must contain the arc of a circle. An example shows that it is not possible to replace "uncountable" by "infinite" in the above theorem. The proof of the theorem is based on the following lemma: If $A$ and $B$ are topological arcs in $R_{2}$ and $A$ contains an infinite number of subarcs which are congruent to $B$, then $B$ is either an arc of a circle or a straight line segment. (Received October 24, 1955.)

\section{Michael Goldberg: Trammel rotors in regular polygons.}

A rotor in a regular polygon is a closed convex curve which remains tangent to all the sides of a fixed $n$-gon during a complete rotation of the curve. Among these, the rotors bounded entirely by arcs of circles have a special interest for several reasons. They are called trammel rotors because of the method of their generation. The rotors of minimal area in the equilateral triangle and in the square have been shown to be trammel rotors and it has been conjectured by the author and others that minimal rotors for other polygons are also trammel rotors [Circular-arc rotors in regular polygons, Amer. Math. Monthly vol. 55 (1948) pp. 393-402]. The author, in the cited paper, gave a method for generating trammel rotors in all regular polygons. These rotors have only one or two axes of symmetry. He now shows how to construct regular trammel rotors of the same circular arcs but with $n-1$ and $n+1$ axes of symmetry. Some of these new rotors have less area than the earlier ones. Fujiwara had already discovered the one for the pentagon [Tôhoku Science Reports vol. 8 (1919) pp. 245246]. More general trammel rotors are obtained by linear combinations of the regular 
rotors. Also, the corresponding rotors in spherical polygons are derived. (Received November 9, 1955.)

244. J. S. Griffin, Jr.: Affine connections in terms of the tangent bundle.

Let $\mathcal{B}\left(B, M, \pi, E^{m}\right)$ be a differentiable bundle of linear spaces, and let $\mathcal{R}=\left(R, M^{\prime}\right.$ $\left.\mu, E^{n}\right)$ and $\mathcal{T}=\left(T, B, \nu, E^{m+n}\right)$ be the tangent bundles. Now $d \pi: T \rightarrow R$, and in fact $\mathcal{Q}=\left(T, R, d \pi, E^{2 m}\right)$ is a bundle of linear spaces, say with operations $\oplus$ and $\circ$. In the particular case that $B=R$, a differentiable retraction $\rho: T \rightarrow T$ which is linear on each fibre $\nu^{-1}(b)$ and whose kernel is that of $d \pi$ defines an affine connection for $M$ if and only if $\rho$ is invariant under these operations: $\rho(x \oplus y)=\rho(x) \oplus \rho(y)$ and $\rho(r \circ x)=r \circ \rho(x)$ for any real number $r$. As to the operations $\oplus$ and 0 , they may be defined in a natural manner from coordinate functions for $\mathcal{Q}$, or in the following intrinsic fashion. Since $\mathbb{B}$ is a bundle of linear spaces for any $r$ there is $\tau_{r}: B \rightarrow B$ defined by $\tau_{r}(x)=r x$, and thus there is $d \tau_{r}: T \rightarrow T$; let $r \circ x=d \tau_{r}(x)$. Let $\left(C, M, \eta, E^{2 m}\right)$ be the Whitney product of $\mathcal{B}$ with itself and let $\left(S, C, \zeta, E^{2 m+n}\right)$ be the tangent bundle; then there are $\xi$, $\iota$, and $\sigma$ on $C$ to $B$ defined by $\xi(x, y)=x+y, \iota(x, y)=x$, and $\sigma(x, y)=y$; these induce $d \xi$, $d \iota$, and $d \sigma$ on $S$ to $T$; one may define $x \oplus y=z$ if there is $v \in S$ such that $d_{\iota}(v)=x, d \sigma(v)=y$, and $d \xi(v)=z$. (Received December 16, 1955.)

245t. H. G. Helfenstein and Max Wyman: Geodesic mapping of minimal surfaces.

In general the only mappings preserving the geodesic lines of a minimal surface onto some other surface are isometries and similarities. There are, however, six classes of exceptional surfaces admitting nontrivial geodesic mappings. They are determined in this paper by means of the theorems of Dini and Lie. Every such surface shares of course this property with all its associates as well as with their similar images; they form an equivalence class. Choosing a simple representative in each equivalence class we obtain a complete system of normal types. Among our surfaces there is a complex one-parameter family of different normal types; in addition there are five other, discrete types. Four of the six cases lead to real minimal surfaces. (Received October 19, 1955.)

\section{L. M. Kelly and Leo Lapidus (p): Metric operations and} Brouwerian geometries. Preliminary report.

If with each two points of a collection, $S$, is associated as the distance between them an element, $u$, of a lattice $L$ with a 0 (the latter serving as the distance between coincident points only), the resulting structure is called a lattice-metrized $(L-M)$ space if, for each three points of the space, the associated distances $u, v$, $w$, satisfy the lattice "triangle inequality," $u \cup v \supset w$. When $S \equiv L$, such an association is called a metric operation, and $L$ is said to be autometrized. When $S \equiv L$ is a Boolean algebra, it is known that symmetric difference is such a metric, yielding a "Boolean geometry." (D. O. Ellis, Autometrized Boolean algebras I and II, Canadian Journal of Mathematics vol. 3 (1951) pp. 87-93 and 145-147.) An analogous symmetric difference function in the more general Brouwerian algebra (logic), gives rise to a "Brouwerian geometry." (E. A. Nordhaus and L. Lapidus, Brouwerian geometry, Canadian Journal of Mathematics vol. 6 (1954) pp. 217-229.) It is shown in the latter paper that the congruence order (L. M. Blumenthal, Distance geometry, Oxford, 1953, pp. 91-92) of a Brouwerian geometry relative to the class of $L-M$ spaces is three iff it is a Boolean geometry. This paper continues the study of Brouwerian geometries. It is shown that the con- 
gruence order of an arbitrary Brouwerian geometry is four. The proof is based on the representation theorem for a distributive lattice as a subdirect union of chains. The implications of the coincidence of metric and lattice betweenness in autometrized lattices is also studied. (Received November 14, 1955.)

247t. E. R. Lorch: Riemannian geometry and smooth convex bodies in Hilbert space.

There is associated with each smooth convex body in Hilbert space a Riemannian geometry defined over that space and which completely characterizes the body. Let $\Omega$ be a convex body defined by $2 G(x) \leqq 1$ where $G(x)$ is positively homogeneous of degree 2 and sufficiently differentiable; furthermore, for the second derivatives we assume $L\|y\|^{2} \leqq G_{y y}(x) \leqq M\|y\|^{2}$. The quadratic form $G_{y y}(x)$ then defines the Riemannian metric. Making use of the fundamental homeomorphism $x \rightarrow \bar{x}=G$. $(x)$ relating $\Omega$ to its polar body $\bar{\Omega}$ (see the author's paper On the volume of smooth convex bodies in Hilbert space, Math. Zeit. vol. 61 (1955) pp. 391-407), the Riemann curvature tensor is calculated. The existence of the Ricci tensor cannot be ascertained due to excessive contraction. The development uses the recent results of Detlef Laugwitz (Differentialgeometrie ohne Dimensionsaxiom, Math. Zeit. vol. 61 (1954) pp. 100-118). In particular his result that the classical ( $n$-dimensional) $i, j, k$ notation can be extended intact to Banach spaces forms the basis of all tensor computations. (Received November 18, 1955.)

\section{8t. T. G. Ostrom: Double transitivity in finite projective planes.}

In this paper, the author proves the following two theorems: Theorem 1. If a finite plane $\pi$, in which $n$ is odd and not a square, is doubly transitive, then $\pi$ is Desarguesian. Theorem 2. If a finite plane $\pi$, in which $n$ is odd and not a square, is doubly transitive for points not on a certain line, then $\pi$ is a Veblen-Wedderburn plane with the line as its special line. Under the restrictions placed on $n$ collineations of order two are perspectivities. A doubly transitive plane contains enough collineations of order two to generate other groups of perspectivities which imply (see R. Baer, Homogeneity of projective planes, Amer. J. Math. vol. 64 (1942) Theorem 6.2) the minor theorem of Desargues. This in turn implies Theorems 1 and 2. (Received November 7, 1955.)

\section{T. K. Pan: Indicatric torsion in a subspace of a Riemannian} space.

A generalization of geodesic torsion of a curve in a surface of an ordinary space has been given and studied by the author in a former paper [Bull. Amer. Math. Soc. Abstract 61-2-335; Proc. Amer. Math. Soc. to appear]. Further generalization of this concept leads to indicatric torsion of a vector field in a subspace $V_{n}$ of a Riemannian space $V_{m}$, which includes as a special case the geodesic torsion of a curve in a $V_{n}$ in a $V_{m}$. Results corresponding to those in the preceding paper are obtained, some new properties are found. Let a vector field $v$ along a curve $C$ in $V_{n}$ in $V_{m}$ consist of unit tangent vectors to the or thogonal trajectories of $C$. The geodesic torsion of $C$ in $V_{n}$ in $V_{m}$, which corresponds to a vector $N$ normal to $V_{n}$, is discovered to be identical with the normal curvature of $v$ along $C$ in $V_{n}$ in $V_{m}$ corresponding to $N$ in a sense as introduced by the author [Amer. J. Math. vol. 74 (1952) pp. 955-966]. (Received November 7, 1955.)

250. C. E. Springer: Union parallel displacement. 
It is known that if a vector with components $\lambda^{\alpha}$ is transported by Levi-Civita parallelism around an infinitesimal circuit in a Riemannian manifold, the change $\Delta \lambda^{\alpha}$ in the components is zero if, and only if, all of the components of the Riemann tensor vanish. In this paper, union parallelism is introduced. The usual components of connection represented by the Christoffel symbols are replaced by components of connection which appear in the differential equations of union curves with respect to a given rectilinear congruence. It is shown that a congruence may be chosen such that if the vector $\lambda^{\alpha}$ is displaced around an infinitesimal circuit in a union parallel manner with respect to this congruence, the vector $\lambda^{\alpha}$ returns to its original position. (Received November 7, 1955.)

251. Saly Ruth Struik: Affine geometry. III. On the affine axiomatic theory of equal area and volume, defined by affine two-space and threespace reflection respectively.

To the 5 groups of axioms conventionally accepted for affine geometry another axiom is added, the two-space affine reflection, to define equal area. Transitivity is included. The three-space affine reflection defines equality of volume, transitivity included. Using reflections addition of triangles and tetrahedrons is defined. Scales of measuring area and volume are reduced to the ratio of parallel vectors. Dehn's proof that compass and ruler fails to produce a regular tetrahedron half the size of a regular one makes it obvious that Hilbert's axiomatic theory of area has no three-space analogon. $\mathrm{He}$ (and Hilbert later in the second edition of the Grundlagen) indicates the necessity of an entirely other approach, e.g. the Cavalieri principle. The reflection does it, though by a definition narrower than the Cavalieri principle. Besides, to demand rigidity when comparing volumes is not in keoping with the spirit of the Erlanger program and would have forestalled, if adhered to, in history, the whole development of integral calculus which so fittingly was initiated by the dire necessity to accurately compare curved barrels of different shape. (Received November 14, 1955.)

252t. R. S. Underwood: Algebraic results obtained by use of extended analytic geometry.

Consider, by way of illustration, the simultaneous equations (1) $x^{2}+y^{2}+z^{2}+u+v$ $=D$ and (2) $x^{2}+y^{2} / 2+z^{2} / 3+u^{2} / 4+v^{2} / 5=1$. Let $X=x^{2}+z^{2}+v$ and $Y=y^{2}+u$, so that the locus of (1) on the $X Y$ plane is the family of lines $X+Y=D$. By this plotting rule and the author's method (Amer. Math. Monthly vol. 61, pp. 525-542) the locus of (2) is found to be a convex area bounded by an ellipse, two parabolas, and a straight line. The slopes of adjacent curves coincide at the junction points. Simple calculation then shows that (1) and (2) have common real solutions only when $-3 \leqq D \leqq 15 / 4$, the sole solutions at the respective end points being $(0,0,0,-4 / 3,-5 / 3)$ and $(0,0, \pm 3 / 2,2 / 3,5 / 6)$. The method is applicable to similar equations in $n$ variables when the first equation has some linear terms, though if it has only one such term families of parabolas instead of lines are used. (Received November 9, 1955.)

253. R. S. Underwood: Some generalizations of theorems about quadric surfaces.

It is known that if $P\left(x_{0}, y_{0}, z_{0}\right)$ is a point on a quadric surface the equation of the plane tangent to the surface at $P$ is obtained by replacing $x^{2}$ by $x_{0} x, x y$ by $\left(x_{0} y+x y_{0}\right) / 2$, etc. If the surface is a hyperboloid of two sheets the tangent plane touches it at one point only, and the two equations have exactly one common real solution. On the 
other hand, a plane tangent to a hyperboloid of one sheet cuts through the surface. The question arises: Does the equation $\sum_{1}^{m} x_{i}^{2} / a_{i}^{2}-\sum_{m+1}^{n} x_{i}^{2} / a_{i}^{2}=1$ have the algebraic properties associated with a hyperboloid of one or two sheets? The answer is: Two sheets when $m=1$ and $n \geqq 3$, and one sheet when $1<m<n$. Moreover, when the first degree equation is plotted as a straight line by the author's method (Amer. Math. Monthly vol. 61, pp. 525-542), the locus of the "quadric surface" becomes the silhouette of a hyperboloid of one or two sheets as the case may be. The one-sheet area-locus may appear as seen from any one of various positions, but in the other case the line clearly touches the locus of the "quadric" at exactly one point. (Received November 9, 1955.)

254t. G. A. Baker, Jr.: A formulation of quantum mechanics based on the quasi-probability distribution induced on phase space.

In a previous paper (Equivalence theorem for quantum mechanics, Bull. Amer. Math. Soc. Abstract 61-3-423) the author proved that a formulation of quantum mechanics based on a quasi-probability distribution is equivalent to the standard Schrödinger one. In this paper the formulation is extended to include measurement. A method is developed whereby, given the quasi-probability distribution representing an ensemble, one may compute the joint distribution of any group of physical quantities. This is a true distribution (no regions of "negative probability") if and only if the physical quantities are simultaneously measurable. In the course of this development, a method is obtained by which, in principle, any quantum mechanical eigenvalue problem may be solved exactly. The great flexibility of this approach is pointed out and the canonical invariance of quantum theory is investigated. Some useful mathematical properties are developed and they are used to introduce some very convenient notation. The framework herein postulated is believed to be complete for purely dynamical systems and to form a clear, logically consistent, self-contained basis for quantum mechanics. In addition, it provides one with an intuitive picture of what the system does in phase space. (Received October 31, 1955.)

255t. G. A. Baker, Jr.: The relation of the quasi-probability distributional representation of quantum mechanics to classical mechanics and certain allied results.

In the limit as Planck's constant, $h$, tends to zero, the formalism of the quasiprobability representation tends to the hamiltonian formalism of classical mechanics. Also approximating differential expressions are developed for key quantities of the theory. From these approximate expressions the conditions under which the interpretation suggested by Bohm (David Bohm, A suggested interpretation of the quantum theory in terms of "hidden" variables. I and II, Phys. Rev. vol. 85 (1952) p. 166) gives results equivalent to those of quantum theory are developed. (Received October 31, 1955.)

256t. G. A. Baker, Jr.: A note on the physical principles underlying the quasi-probability distribution for quantum mechanics.

The point distribution in phase space of Newtonian mechanics satisfies the equatiln $f=\left(0^{+}\right) f f$. In quantum mechanics one seeks to smear out the classical point. This alteration is accomplished by replacing $\left(0^{+}\right)$by Planck's constant, $h$, and $f f$ by the product of $f$ with itself with respect to a particular non-associative multiplication. With the aid of this change of the classical concept of a representation in terms of a 
single moving phase point, and Bohr's correspondence principle, the derivation of an equation equivalent to Schrödinger's wave equation follows. The effect of a measurement on a quantum mechanical system is seen to be the placement of the system in a state such that the expected value is an extremum in the sense of the calculus of variations with respect to the class of quasi-probability functions. The measured quantity may assume only these extreme values. These considerations suffice to establish the postulates of the quasi-probability distributional representation described in the preceding papers. (Received October 31, 1955.)

\section{7t. Hugo Ribeiro: Universal completeness for relational systems.}

For terminology see A. Tarski, Contributions to the theory of models, I and II, Proc. Dutch Acad. Sci. Ser. A, vol. 57, no. 5, and vol. 58, no 1. Equational completeness for abstract algebras has been defined by Kalicki and Scott and studied also by Tarski, Bull. Amer. Math. Soc. Abstracts 58-6-582, 59-1-116, 59-1-119, 60-2-202. A set, $\Sigma$, of sentences of the formalized theory $T(R)$ constructed for a given similarity class, $R$, of relational systems is said to be universally complete if and only if for any universal sentence $\phi$, of $T(R)$, either every model of $\Sigma$ is a model of $\phi$ or there is a universal sentence, $\alpha$, involving only the identity symbol, together with the other logical constants, such that every model of $\Sigma$ is a model of $\phi$ if and only if it is a model of $\alpha$. The set of sentences defining the class of all systems $\langle A, R\rangle$ where $A$ is a nonvoid set and $R$ is a binary relation which orders $A$ linearly is universally complete. (Received November 14, 1955.)

\section{8t. H. D. Sprinkle: $A$ primitive recursive foundation for random-} ness. Preliminary report.

Recursive function theory is used to define the notion of an element being random with respect to certain sequences (probability collectives). The main theorem proves the existence of a probability collective (of any finite cardinal) such that each element of it is random with respect to the collective. The proof is constructive if there is an effective well-ordering of a certain subclass of primitive recursive functions. (Received November 7, 1955.)

\section{Statistics ANd Probability}

259. G. E. Baxter and M. D. Donsker ( $\mathrm{p})$ : On the zeros of the sample functions of certain gaussian processes.

Let $\{y(s), 0 \leqq s<\infty\}$ be the Wiener process. For each fixed $t>0$, the integral $x(t)=\int_{0}^{\infty} e^{-s t} d y(s)$ exists with probability one, and the process, $\{x(t), 0<t<\infty\}$, is Gaussian with zero mean function and covariance function $\left(t_{1}+t_{2}\right)^{-1}$. The sample functions $x(t)$ are analytic with probability one. This paper is principally concerned with the question: what is the expected number of crossings that the sample functions $x(t)$ make with a curve $r(t)$ on a given interval $(a, b)$ ? For analytic $r(t)$ an explicit answer is obtained. The corresponding result is derived for Gaussian processes obtained from the Wiener process by the transformation $z(t)=\int_{0}^{\infty} e^{-g(t) s} d y(s)$ where $g(t)$ is analytic. The following theorem is also obtained: let $f(t)$ be an increasing continuous function on $(a, \infty)$ vanishing at $a>0$. The Gaussian process, $\{z(t), a \leqq t<\infty\}$, whose mean function is zero and whose covariance function is $\left(e^{2 \pi f\left(t_{1}\right)}+e^{2 \pi f\left(t_{2}\right)}\right)^{-1}$ has exactly $f(T)$ as its expected number of zeros on the interval $(a, T)$ for every $T$. The preceding results are used to obtain certain strong limit theorems on the asymptotic behavior of the sample 
functions of Gaussian rocesses obtained from the Wiener process by the transformations above. (Received November 14, 1955.)

\section{0t. S. S. Cairns: Balance scale sorting.}

Given (1) a set $W$ of $n$ objects, indistinguishable save that the members of a subset $H$ of $h$ objects are slightly heavier than the rest, (2) a balance scale, one seeks weighing programs minimizing either [Problem $M(n, h)$ ] the maximum number of weighings which may be required to cull out $H$ or [Problem $E(n, h)]$ the expected number of such weighings. Problem $M(n, 1)$ is a familiar puzzle. Problem $E(n, 1)$ is here solved, under various hypotheses. Problem $M(n, 2)$ is partially solved. The methods and results may serve as a basis for more extended work on Problems $M(n, h)$ and $E(n, h)$. Some of the techniques, moreover, involve manipulations of series which may prove useful in other combinatorial problems. (Received November 14, 1955.)

\section{1t. K. L. Chung: Some new developments in Markov chains.}

The new concept of a post-exit process imbedded in a continuous parameter Markov chain is introduced. It is shown that this process has the same transition probabilities as the original chain but its initial distribution is of a novel kind. An imbedded renewal process is also studied. Together they yield various relations implying the differentiability of the transition probability functions and certain generalized Kolmogorov differential equations. (Received November 15, 1955.)

262t. J. C. Kiefer and Jacob Wolfowitz: Consistency of the maximum likelihood estimator in the presence of infinitely many incidental parameters.

The authors show, under usual regularity conditions, that the maximum likelihood estimator of a structural parameter is strongly consistent, when the (infinitely many) incidental parameters are independently distributed chance variables with a common unknown distribution function. The latter is also consistently estimated although it is not assumed to belong to a parametric class. Application is made to several problems, in particular to the problem of estimating a straight line with both variables subject to error, which thus after all has a maximum likelihood solution. (Received September 12, 1955.)

263. Klaus Krickeberg: Convergence of nondenumerable martingales. Preliminary report.

Since a Boolean $\sigma$-algebra $\mathfrak{B}$ with unity $E$ bearing a finite strictly positive measure $\mu$ and the vector lattice $\mathfrak{F}$ of all $\mathfrak{B}$-measurable real functions on $E$ are complete, every Moore-Smith sequence $\left(f_{\sigma} ; \sigma \in \Theta\right)$ in $\mathfrak{F}$, with an index set $\Theta$ directed by $\ll$, has an upper and lower limit in $\mathfrak{F}$. In a previous paper of the author the usual theorems on interchanging integral and limits have been generalized to the present case, and criteria for $\int_{E}\left(\lim \sup f_{\sigma}\right) d \mu \leqq \lim \sup \int_{E} f_{\sigma} d \mu$ derived. An application yields convergence theorems on martingales with index set $\Theta$. The sequence $\left(f_{\sigma}\right)$ is called a martingale relative to a monotone increasing sequence $\left(\mathfrak{B}_{\sigma} ; \sigma \in \Theta\right)$ of Boolean $\sigma$-subalgebras of $\mathfrak{B}$, if $f_{\sigma}$ is $\mathfrak{B}_{\sigma}$-measurable and summable over $E$, and $\int_{A} f_{\sigma} d_{\mu}=\int_{A} f_{\tau} d_{\mu}$ for $A \in \mathfrak{B}_{\tau}$ and $\tau \ll \sigma$. For fixed $\left(\mathfrak{B}_{\sigma}\right)$, the space of all martingales relative to $\left(\mathfrak{B}_{\sigma}\right)$ is a complete vector lattice. $\left(\mathfrak{B}_{\sigma}\right)$ is said to enjoy Vitali's property, if for any $\epsilon>0$, any $A \in \mathfrak{B}$, and any sequence $\left(K_{\sigma} ; \sigma \in \Theta\right)$ with $K_{\sigma} \in \mathfrak{B}_{\sigma}$ and $A \leqq \bigvee\left\{K_{\sigma} ; \tau \ll \sigma\right\}$ for every $\tau$, there exist a 
finite subset $\left\{\xi_{1}, \cdots, \xi_{r}\right\}$ of $\Theta$ and disjoint $L_{i} \in \mathfrak{B}_{\xi_{i}}, i=1, \cdots, r$, such that $L_{i} \leqq K_{\xi}$ and $\mu\left(A-\left(A \wedge \bigvee L_{i}\right)\right)<\epsilon$. Under this assumption many theorems known before only in the case $\Theta=(1,2, \cdots)$ still hold, e.g. convergence of $\left(f_{\sigma}\right)$, if $\sup \int_{E}\left|f_{\sigma}\right| d \mu<+\infty$. Vitali's condition is indispensable here, as shown by examples, but is satisfied, if $\ll$ is a linear order. (Received November 10, 1955.)

264t. J. M. Shapiro: Convergence of moments of sums of truncated random variables.

For $n=1,2, \cdots$ let $S_{n}=x_{n_{1}}+\cdots+x_{n k_{n}}$ be the sum of independent, infinitesimal, random variables, and let $F_{n}(x)$ be the distribution function of $S_{n}$. Assume (A): there exists an $a>0$ such that $F_{n k}(x)=0$ for $x \leqq-a$ and $F_{n k}(x)=1$ for $x \geqq a$ where $F_{n k}(x)$ is the distribution function of $x_{n b}$. In a previous paper (Bull. Amer. Math. Soc. Abstract 61-3-435) the author has shown that if $F_{n}(x)$ converges to a limiting distribution $F(x)$ then $F(x)$ has finite moments of all orders. Assuming $F_{n}(x) \rightarrow F(x)$ it is shown that for each positive integer $k$ the $k$ th moment of $F_{n}(x)$ approaches the $k$ th moment of $F(x)$ as $n \rightarrow \infty$. If the assumption (A) is not made let $x_{n k}^{a}=x_{n k}$ if $\left|x_{n k}\right|<a$, $x_{n k}^{a}=0$ otherwise, and let $F_{n}^{a}(x)$ be the distribution function of $S_{n}^{a}=x_{n 1}^{a}+\cdots+x_{n k_{n}}^{a}$. Using the results of the above paper conditions are given for the existence of a function $F^{a}(x)$ such that the $k$ th moment of $F_{n}^{a}(x)$ approaches the $k$ th moment of $F^{a}(x)$ and the form of $F^{a}(x)$ is also discussed. (Received November 14, 1955.)

\section{TOPOLOGY}

\section{L. W. Anderson: Cutpoints in topological lattices.}

A topological lattice is a triple $(L, \wedge, \vee)$ where $L$ is a Hausdorff space and $\bigwedge: L \times L \rightarrow L$ and $\bigvee: L \times L \rightarrow L$ are continuous functions satisfying the usual conditions stipulated for a lattice. It is shown that if $L$ is a connected topological lattice and if $a \in L$ then $a$ is related to every element of $L$ (i.e. $a \leqq x$ or $x \leqq a$ for all $x \in L$ ) if, and only if, either $a=0$ or $a=1$ or $a$ is a cutpoint of $L$. It follows from this result that (1) a connected topological lattice has at most two end points, (2) a connected topological chain with 0 and 1 is irreducibly connected about 0 and 1 . (Received November $15,1955$.

\section{R. D. Anderson: Atomic decompositions of continua.}

An upper semi-continuous decomposition $G$ of a continuum $M$ into continua is said to be atomic provided that if $K$ is any subcontinuum of $M$ intersecting two elements of $G$, then $K$ contains each of the elements of $G$ which it intersects. The author shows that if $S$ is any continuum (compact, metric, connected set) and $n$ is any positive integer, then there exist a continuum $M$ and a monotone open mapping $f$ of $M$ onto $S$ such that the collection of inverses of the points of $S$ under $f$ is atomic, and each of $M$ and the various inverse sets under $f$ contains an $n$-cell and is of dimension $n$. The conclusion may be altered to assert that each of $M$ and the inverse sets contains a homeomorphic image of the Hilbert cube. The argument used to establish this result is a modification of the author's proof of the existence of monotone interior dimension raising mappings (Duke Math. J. vol. 19 (1952) pp. 359-366). (Received November 14, 1955.)

267t. R. D. Anderson and Gustave Choquet: Plane continua, no two nondegenerate subcontinua of which are homeomorphic.

The authors give a construction of a plane continuum such that no two subcon- 
tinua of it are homeomorphic to each other. The continuum cited has the additional properties that (1) No subcontinuum of it separates the plane, (2) It does not contain uncountably many mutually exclusive non-degenerate subcontinua, and (3) Each non-degenerate subcontinuum admits an irreducible monotone mapping onto a continuum which is the sum of finitely many arcs, each pair having exactly an end point of all in common. A somewhat similar alternative construction is suggested yielding such a continuum having the property, in lieu of (1) and (3) above, that every nondegenerate subcontinuum of it separates the plane. (Received November 14, 1955.)

\section{R. W. Bagley: Transfinite sequential order topology.}

It is shown that transfinite sequences are not adequate to describe the topology of pointwise convergence on $2 R$ (taking the discrete topology on $\{0,1\}$ ) when $R$ is uncountable. Here, "transfinite sequence" means a function on a well ordered set into a set. This result holds for the Tychonoff cube as well as any direct product of lattices which contains $2 R$ as a sublattice. In each case it is assumed that there are uncountably many coordinate spaces. The transfinite sequential order topology on a complete lattice is defined in a manner analogous to the sequential order topology and the order topology (G. Birkhoff, Lattice theory, rev. ed., Amer. Math. Soc. Colloquium Publications, 1948). The above result follows from the fact that, when $R$ is uncountable, the transfinite sequential order topology is properly less than the order topology and properly greater than the sequential order topology. The order topology coincides with the topology of pointwise convergence (O. Frink, Topology in lattices, Trans. Amer. Math. Soc. vol. 51 (1942) pp. 569-582 and J. W. Tukey, Convergence and uniformity in topology, Princeton, 1940). (Received November 14, 1955.)

\section{Lida K. Barrett: On a question raised by $R$. H. Bing.}

R. H. Bing in Partitioning continuous curves (Bull. Amer. Math. Soc. vol. 58 (1952) p. 541) raises the following question, which if answered in the affirmative would give an alternate method of partitioning continuous curves. Question: Does there exist a positive integer $n$ such that the following holds for each continuous curve $M$, each positive number $e$, and each pair of mutually exclusive closed subsets $H$ and $K$ of $M$ ? If $R$ is a finite subset of $M$ such that each point of $R$ belongs to an arc in $M$ of diameter less than $e$ that intersects $H+K$, there are two collections $A_{H}$ and $A_{k}$ of arcs satisfying the following conditions: (a) Each element of $A_{H}$ intersects $H$ but not $K$ and each element of $A_{K}$ intersects $K$ but not $H$ nor any element of $A_{H}$. (b) Each element of $R$ belongs to an element of $A_{H}+A_{K}$. (c) Each element of $A_{H}+A_{K}$ is of diameter less than $n e$. Bing gives an example composed of two arcs to show that the question cannot be answered in the affirmative for $n=1$. The present paper gives an example composed of twenty-one arcs to show that it cannot be answered in the affirmative for $n=2$. (Received November 4, 1955.)

\section{R. H. Bing: An alternate proof that 3-manifolds can be triangu- lated.}

The author's approximation theorem for surfaces is extended to apply to topological 2-complexes. The approximation theorem for 2-complexes may be stated as follows. If in $E^{3}, C$ is a topological 2-complex and $f$ is a nonnegative continuous function defined on $C$, then there is a topological 2-complex $C^{\prime}$ and a homeomorphism $h$ of $C$ onto $C^{\prime}$ such that $h$ moves no point $x$ of $C$ by more than $f(x)$ and $C^{\prime}$ is locally polyhedral at $h(x)$ if $f(x)>0$. This theorem has interesting applications when applied to the 2skeleton of a triangulation of an open subset $U$ of $E^{3}$. It shows that if $f$ is a continu- 
ous nonnegative function on $U$ and $h$ ia a homeomorphism of $U$ into $E^{3}$, then there is a homeomorphism $h^{\prime}$ of $U$ into $E^{3}$ such that $\rho\left(h(x), h^{\prime}(x)\right) \leqq f(x)$ and $h^{\prime}$ is piecewise linear at $x$ if $f(x)>0$. It may likewise be used to show that each 3-manifold can be triangulated. These last two results are due to Moise who proved them by other methods. (Received November 14, 1955.)

271. A. H. Copeland, Jr.: The Whitehead bracket product on $C W$ complexes.

Suppose $X, N, M$ are CW-complexes with $X$ being 1-connected, $N, n$-connected, and $M, m$-connected $(1<n \leqq m), \pi_{n}(N) \approx \pi_{n}(X), \pi_{m}(M) \approx \pi_{m}(X)$. Let $Y \supset X$ be a realization of the $(n+m-1)$ Postnikov complex associated with $X$, and $k \in H^{n+m}(Y$; $\left.\pi_{n+m-1}(X)\right)$ the Postnikov invarient. In addition, suppose that there are maps $\xi: N \rightarrow Y, \eta: M \rightarrow Y$ such that the induced homomorphisms $\xi_{\sharp}: \pi_{n}(N) \rightarrow \pi_{n}(Y), \eta_{\sharp}: \pi_{m}(M)$ $\rightarrow \pi_{m}(Y)$ are isomorphisms. The maps $\xi, \eta$ give rise to a map $\theta: N \times M \rightarrow Y$. The element $\theta^{*} k \in H^{n+m}\left(N \times M ; \pi_{n+m-1}(X)\right)$ defines an element $W \in \operatorname{Hom}\left(\pi_{n}(X) \otimes \pi_{m}(X)\right.$; $\left.\pi_{n+m-1}(X)\right)$ such that $W(\alpha \otimes \beta)=[\alpha, \beta]$ for $\alpha \in \pi_{n}(X), \beta \in \pi_{m}(X)$. In some cases this construction may be used to give an effective procedure for finding bracket products on $X$. (Received November 14, 1955.)

\section{M. L. Curtis: A spectral sequence associated with a topological} space.

The singular chain group $C(X)$ of a topological space $X$ can be given a decreasing filtration by letting $C^{p}(X)$ be the subgroup of chains of $C(X)$ which are obtainable from the $p$-iterated loops by a transgression on chains. This filtration gives rise to a certain exact couple which, in turn, gives rise to a spectral sequence. The term $E_{\infty}$ consists of images of $\pi_{k}(X)$ in $H_{k}(X)$. The term $E_{1}$ can be interpreted as follows. Let $K$ be the total singular complex of $X$. There exists a sequence of subcomplexes $K=K_{0} \supset K_{1} \supset K_{2} \supset K_{3} \supset \ldots$ such that $E_{1}$ is the direct sum of the relative homology groups $H_{r}\left(K_{m}, K_{m+1}\right)$. Instead of the total singular complex one may use a minimal subcomplex of $K$. (Received November 14, 1955.)

273. M. L. Curtis and R. L. Wilder (p): The existence of certain types of manifolds.

The first part of the paper gives a class of polyhedral 3-manifolds $M$, each separating the 4-sphere $S^{4}$ and with homology groups $H_{1}(M)\left(=H_{2}(M)\right)=0$, but with $\pi_{1}(M) \neq 0$; thus some "Poincaré spaces" are imbeddable in $S^{4}$. Also, given any finitely-generated abelian group $G$, there exists a 3-manifold $M$ in $S^{4}$ with $H_{1}(M)=G$. In the second part of the paper a negative answer is found for the question: Since the metric cases of the 2 -gcms reduce to the classical types, and the 3 -gcms generally do not, can one state that a sphere-like $3-\mathrm{gcm}$ in euclidean space $E^{4}$ must be a classical manifold? 3-gcms are constructed in $E^{4}$ which have the same homology and homotopy character as the 3-sphere (globally and locally), but which are not locally euclidean. In the third part of the paper a negative answer is found for a question proposed by Griffiths (Michigan Mathematical Journal vol. 2 (1953) pp. 61-89, Section 6.19) by showing the existence of a 3-dimensional "homotopy manifold" which is not locally euclidean (this is precisely a space previously obtained by R. H. Bing by identifying the points on a certain set of tame arcs in $S^{3}$ ). (Received November 14,1955.)

274. W. F. Davison: Convergent sequences and mosaics.

Certain topological spaces are investigated, wherein sequences are the principal 
associated convergence objects. A mosaic of compact metric spaces is a collection $\left\{\left(X_{a}, T_{a}\right): a \in A\right\}$ such that each $\left(X_{a}, T_{a}\right)$ is compact metric, and $E$ being $T_{a}$-closed implies $E \cap X_{b}$ is $T_{b}$-closed for $a, b \in A$. The mosaic topology $T$ on $X=\bigcup\left\{X_{a}: a \in A\right\}$ is obtained by $E$ being $T$-closed provided $E \cap X_{a}$ is $T_{a}$-closed for all $a \in A$. In mosaic spaces the following hold: (i) limits of $T$-convergent sequences are unique; (ii) the topology derived from the $T$-convergent sequences is $T$ itself; (iii) countable compactness is equivalent to sequential compactness; and (iv) countably compact sets are closed. A space satisfying (i) and (ii) is the mosaic space of some mosaic of compact metric spaces. In mosaic spaces open sets and closed sets are mosaic spaces in their relative topology, and the following are equivalent: (1) every subspace is a mosaic space; (2) a limit point of a set is the limit of some sequence in the set; (3) $\left\{x_{n m}\right\}$ converging to $x_{n}$ and $\left\{x_{n}\right\}$ converging to $x$ imply a sequence in the set of $\left\{x_{n m}\right\}$ converges to $x$; and (4) sequence closure is topological closure. Finally the construction of mosaic spaces is generalized, and a non-Hausdorff mosaic space is exhibited. (Received November 14, 1955.)

\section{Eldon Dyer: Open mappings and regular convergence.}

If $G$ is a collection of compact subspaces of a compact metric space $X$, of denotes the metric space in which the points are the elements of $G$ and the metric is the Hausdorff metric between elements of $G$. J. H. Roberts has shown (Duke Math. J. vol. 2 (1936) pp. 10-19) that if $G$ is a continuous collection of mutually exclusive arcs filling $X$, there is a subcollection $G_{1}$ of $G$ such that $B_{1}$ is a dense $G_{\delta}$ subset of $\&$ and every element $g$ of $G_{1}$ is approached 0-regularly by every sequence of elements of $G$ converging to $g$. In the present paper it is shown that if $n$ is a non-negative integer and $G$ is a collection of compact $1 c^{n}$ subspaces of $X$ such that $(5)$ is a topologically complete space, then there is a sub-collection $G_{1}$ of $G$ such that $B_{1}$ is a dense $G_{\delta}$ subset of \&) and every element $g$ of $G_{1}$ is approached $n$-regularly by every sequence of elements of $G$ converging to $g$. It is also shown that if $f$ is an open mapping of an open subset of an $n$-sphere into a metric space $M$ such that for each point $x$ of $M, f^{-1}(x)$ is compact, $1 c^{n}$, and homologically trivial, then $f$ is a homeomorphism. The coefficient group is any field or elementary compact group. (Received November 14, 1955.)

\section{J. G. Horne, Jr.: On o-ideals in $C(X)$.}

We study $o$-ideals of Milgram in a class of sub-semigroups of $C(X) \quad(=$ semigroup under multiplication of all continuous complex functions on the topological space $X$ ) which Civin and Yood have called Silov semigroups. In a commutative ring $\left(R,+,^{\prime}\right)$ with unit, a semigroup $S C\left(R,{ }^{\prime}\right)$ is Silov if and only if three elements $e_{1}, e_{2}, e_{3}$, in $R$, related by the equations $e_{2} e_{1}=e_{1}$ and $e_{2} e_{3}=e_{2}$, yield an $e_{2}^{\prime} \in S$ such that $e_{2}^{\prime} e_{1}=e_{1}$ and $e_{2}^{\prime} e_{3}=e_{2}^{\prime}$ hold. For such semigroups $S$ we have the equivalence of (1) $I$ is an $o$-ideal in $S$, and (2) there is an $o$-ideal $I^{\prime}$ of $\left(R,,^{\prime}\right)$ such that $I=I^{\prime} \cap S$. Further, the correspondence $I^{\prime} \rightarrow I^{\prime} \cap S$ is one-to-one. In the case $\left(R,{ }^{\prime}\right)=\left(C(X),{ }^{\prime}\right)$ then (1) and (2) are equivalent to (3) there exists a completely regular filter $g(I)$ of open sets in $X$ such that $I=\left\{f \in S ; Z_{f} \supset G\right.$, for some $\left.G \in g(I)\right\} . I$ is a maximal if and only if $g(I)$ is a completely regular end. A completely regular filter $G$ of open sets is an ideal in the lattice of open sets such that for each $G_{1} \in G$, there is a $G \in G$ and a continuous real function $e$ which vanishes on $G_{0}$ and is one outside $G_{1}$. (Received November 14,1955.)

\section{I. S. Krule: Structs on the 1-sphere.}

Let $X$ be the 1-sphere and let $L$ be a reflexive monotone continuous struct on $X$. (For terminology see A. D. Wallace, Struct ideals, Proc. Amer. Math. Soc. vol. 6 
(1955) pp. 634-638.) For $A \subset X$ let $L \cap(A \times A)$ be called the $L$-induced relation on $A$. Let $G_{L}=\{x \in X: L(x)=X\}$. The following result is proved: If $x \in K_{L}$ then $L_{x}=x$ or $L_{x}=K_{L}$. If $X \neq K_{L}$ then (1) $G_{L}$ consists of exactly one point; (2) if $C_{1}$ and $C_{2}$ are the components of $X-\left(K_{L} \cup G_{L}\right)$ then $L\left(C_{1}\right) \cap C_{2} \neq \square \neq C_{1} \cap L\left(C_{2}\right)$; (3) if $x \in X-K_{L}$ then $L_{x}=x$ or $L_{x}$ consists of exactly two points, one in $C_{1}$ and one in $C_{2} ;(4)$ if $R_{i}$ is the $L$-induced relation on $\bar{C}_{i}$ then $\bar{C}_{i}$ is an ordered space with respect to $R_{i}(i=1,2) ;(5)$ if $x \in \overline{\left(X-K_{L}\right)}$ then $\sigma L(x)$ is connected; (6) $G_{L} \cup\left\{x \in X: L_{x} \neq x\right\}$ is closed; (7) the quotient space $X /(L \cap \sigma L)$ is a one-dimensional cyclic chain which is topologically embeddable in the plane. (Received November 10, 1955.)

278. P. S. Mostert (p) and A. L. Shields: On semigroups on a manifold.

Let $S$ be a topological semigroup with identity 1 . Theorem. If there is a neighborhood of 1 homeomorphic to a Euclidean space, then there is an open, connected Lie subgroup of $S$ containing 1. Corollary. Let $(S, B)$ be a compact relative manifold with boundary (i.e., $B$ is a closed subset of $S$ and $S-B$ is locally Euclidean). If $S$ is a topological semigroup with identity, then, if $H(1)$ denotes the maximal subgroup of 1 , either $H(1)=S$, or $H(1) \subset B$. (Received November 14, 1955.)

\section{9t. C. D. Papakyriakopoulos: On a lemma of Kneser.}

The following theorem is proved: Let $M$ be a 3-manifold, compact or not, with boundary $N$ formed by a number $(>0, \leqq \infty)$ of surfaces closed or not. Let $L$ be a loop belonging to an open set $U$ of an orientable component $N^{\prime}$ of $N$ such that $L \simeq 0$ in $M$, and not $\simeq 0$ on $N$ (the symbol $\simeq$ means homotopic to $)$. Then there exists a simple ( $=$ without multiple points) loop $L_{0}$ in $U$ such that $L_{0} \simeq 0$ in $M$, and not $\simeq 0$ on $N$. The first to study problems of this sort was $\mathrm{H}$. Kneser (Geschlossene Flächen in dreidimensionalen Mannigfaltigkeiten, Jber. Deutschen Math. Verein vol. 38 (1929) pp. 248-260, Hilfssatz of p. 248). J. H. C. Whitehead proved an important special case of the theorem (On doubled knots, J. London Math. Soc. vol. 12 (1937) pp. 63-71, lemma of p. 65). The proof of the theorem makes use of Whitehead's result. A consequence of the theorem is a negative answer to a problem of Bing (Summary of Lectures and Seminars, Summer Institute on Set Theoretic Topology, Madison, Wisconsin (1955) p. 57, problem 12). (Received November 4, 1955.)

280. B. J. Pearson: $A$ connected point set in the plane which spirals down on each of its points.

Let $A B C$ be a triangle in the plane and $G$ the collection of all straight line interval ${ }^{\mathrm{s}}$ with one end point at $A$ and the other end point on the interval $B C$. If $G^{\prime}$ is a collection of arcs in the plane such that (1) each arc of $G$ is a proper subarc of some arc of $G^{\prime}$, (2) each arc of $G^{\prime}$ has one end point at $A$ and contains some arc of $G$, and (3) no two arcs of $G^{\prime}$ have any point in common except $A$, then $G^{\prime}$ is said to be an extension of $G$. It is shown that there exist an extension $G^{\prime}$ of $G$ and a compact, connected inner limiting set $M$ not containing $A$ which is connected im kleinen at all but a countable number of its points such that (1) each point of $M$ is an end point of some arc of $G^{\prime}$, (2) each arc of $G^{\prime}$ has one end point in $M$, and (3) each arc of $G^{\prime}$ spirals down on the point of $M$ belonging to it but does not spiral down on any other point. (Received November 14, 1955.)

281t. J. P. Roth: A combinatorial topological method for the synthesis of switching systems in $n$ variables. 
The switching circuit problem, arising e.g. in the design of telephone switching systems, computing machines, and control systems, has previously been treated from the viewpoint of symbolic logic, notably by Shannon and Quine. There exists no effective mechanical algorithm for finding circuits performing prescribed functions and having a minimum number of switches. Here the problem is considered to define a cubical complex; a routine, "fast" mechanical process is given which automatically gives a switching system in $n$ variables which has a minimum number of switches over a very restricted class of series-parallel circuits. Other operations are defined which permit further reduction of the system, allowing for bridge circuits, etc. But no general procedure has yet been found to obtain the absolute minimum. (Received November 15, 1955.)

282. R. F. Williams: Concerning dimension raising mappings of cells.

It is shown that if $n$ and $m$ are integers, $m>n>1$, and $f$ is a mapping of an $n$-cell $I^{n}$ into an $m$-cell $I^{m}$, then there exists a sub-continuum of $I^{n}$ whose image under $f$ is of dimension $\leqq m-2$. The proof is based on a category argument in the space of subcontinua of $I^{n}$ and the existence of certain (familiar) $m-2$ dimensional continua in $I^{m}$. (Received November 14,1955 .)

J. W. T. Youngs, Associate Secretary 